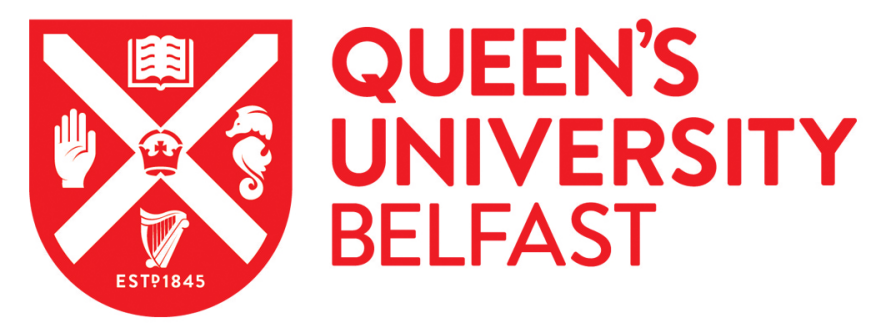

\title{
Local form-subordination condition and Riesz basisness of root systems
}

Mityagin, B., \& Siegl, P. (2019). Local form-subordination condition and Riesz basisness of root systems. Journal d'Analyse Mathématique. https://doi.org/10.1007/s11854-019-0053-3

\section{Published in:}

Journal d'Analyse Mathématique

\section{Document Version:}

Peer reviewed version

Queen's University Belfast - Research Portal:

Link to publication record in Queen's University Belfast Research Portal

Publisher rights

(c) 2017 Hebrew University Magnes Press.

This work is made available online in accordance with the publisher's policies. Please refer to any applicable terms of use of the publisher.

\section{General rights}

Copyright for the publications made accessible via the Queen's University Belfast Research Portal is retained by the author(s) and / or other copyright owners and it is a condition of accessing these publications that users recognise and abide by the legal requirements associated with these rights.

Take down policy

The Research Portal is Queen's institutional repository that provides access to Queen's research output. Every effort has been made to ensure that content in the Research Portal does not infringe any person's rights, or applicable UK laws. If you discover content in the Research Portal that you believe breaches copyright or violates any law, please contact openaccess@qub.ac.uk. 


\title{
LOCAL FORM-SUBORDINATION CONDITION AND RIESZ BASISNESS OF ROOT SYSTEMS
}

\author{
BORIS MITYAGIN AND PETR SIEGL
}

In memory of our colleague and friend Michael Solomyak.

\begin{abstract}
We exploit the so called form-local subordination in the analysis of non-symmetric perturbations of unbounded self-adjoint operators with isolated simple positive eigenvalues. If the appropriate condition relating the size of gaps between the unperturbed eigenvalues and the strength of perturbation, measured by the form-local subordination, is satisfied, the root system of the perturbed operator contains a Riesz basis and usual asymptotic formulas for perturbed eigenvalues and eigenvectors hold. The power of the abstract perturbation results is demonstrated particularly on Schrödinger operators with possibly unbounded or singular complex potential perturbations.
\end{abstract}

\section{INTRODUCTION}

Let $T$ be a Schrödinger operator in $L^{2}(\mathbb{R})$

$$
T=-\frac{\mathrm{d}^{2}}{\mathrm{~d} x^{2}}+Q(x)+V(x)
$$

where $Q$ is a sufficiently regular real single-well potential behaving as $|x|^{\beta}, \beta \geq 1$, at infinity and $V$ is a complex, possibly unbounded or singular, perturbation. The spectrum of $T$ is discrete under mild restrictions on $V$, guaranteeing basically that $V$ is indeed a "small" perturbation of the self-adjoint operator

$$
A=-\frac{\mathrm{d}^{2}}{\mathrm{~d} x^{2}}+Q(x)
$$

However, it is quite unclear under which conditions on $V$ the eigensystem of $T$ shares the good properties of the eigenfunctions of $A$, forming an orthonormal basis of $L^{2}(\mathbb{R})$. More specifically, for which $V$ does the eigensystem of $T$ contain a Riesz basis?

Notice that the Riesz basisness of eigensystem is particularly strongly linked with the spectral stability/instability (pseudospectra/resolvent norm far from spectrum) of the spectrum of $T$. The spectral instability is well-known to occur for Schrödinger operators with complex potentials like the rotated oscillator of Davies

$$
-\frac{\mathrm{d}^{2}}{\mathrm{~d} x^{2}}+\mathrm{i} x^{2}
$$

for which the eigensystem does not contain even a basis. Such results are obtained in several works and are typically based on the norm estimates of the resolvent (pseudospectra) or eigenprojections, see e.g. [5, 6, 7, 18, 19, 20, 36, 23]. Nonetheless,

Date: 14 th March 2017.

2010 Mathematics Subject Classification. 47A55, 47A70, 34L10, 34L40.

Key words and phrases. non-self-adjoint operators, Riesz basis, Schrödinger operators with complex and singular potentials.

We acknowledge the support of the University of Bern (B.M., October-November 2015) and the Ohio State University (P.S., May 2016) for our visits there. The research of P.S. is supported by the Swiss National Science Foundation Ambizione grant No. PZ00P2_154786. 
by proving the unboundedness the eigenprojection norms in [30], one shows that the eigensystem for the shifted oscillator

$$
-\frac{\mathrm{d}^{2}}{\mathrm{~d} x^{2}}+x^{2}+\mathrm{i} x
$$

contains no basis also; moreover, let us notice that $Q(x)=x^{2}$ clearly dominates the imaginary perturbation $V(x)=\mathrm{i} x$. On the other hand, it was showed in [29] that the eigensystem of

$$
-\frac{\mathrm{d}^{2}}{\mathrm{~d} x^{2}}+x^{2}+\mathrm{i} \delta(x)
$$

does contain a Riesz basis; the latter holds also for an infinite number of $\delta$ 's, namely for $\sum_{k \in \mathbb{Z}} \nu_{k} \delta\left(x-x_{k}\right)$ with $\nu \in \ell^{1}(\mathbb{Z})$ and $\left\{x_{k}\right\} \subset \mathbb{R}$, see $[29,26,27,28,30]$. The perturbations in (1.4) and (1.5) seem to be of a different nature, nevertheless, their strength is the same if described in classical terms of relative boundedness or $p$ subordination; see e.g. $[22,24,21]$ for these notions. More specifically, when viewed in the sense of quadratic forms,

$$
b_{1}[\psi] \equiv b_{\mathrm{i} x}[\psi]=\mathrm{i} \int_{\mathbb{R}} x|\psi(x)|^{2} \mathrm{~d} x, \quad b_{2}[\psi] \equiv b_{\mathrm{i} \delta(x)}[\psi]=\mathrm{i}|\psi(0)|^{2},
$$

both $b_{1}$ and $b_{2}$ are $p$-subordinated with $p=1 / 2$ to the form $a+1$, associated with the self-adjoint harmonic oscillator (with an inessential shift by 1 ). Namely, there is $C>0$ such that, for all $\psi \in \operatorname{Dom}(a)=\left\{\psi \in W^{1,2}(\mathbb{R}): x \psi(x) \in L^{2}(\mathbb{R})\right\}$,

$$
\left|b_{i}[\psi]\right| \leq C\left(a[\psi]+\|\psi\|^{2}\right)^{p}\|\psi\|^{2(1-p)}, \quad i=1,2, \quad p=\frac{1}{2},
$$

where

$$
a[\psi]=\left\|\psi^{\prime}\right\|^{2}+\int_{\mathbb{R}} x^{2}|\psi(x)|^{2} \mathrm{~d} x .
$$

These two examples clearly show that the classical sufficient conditions for the Riesz basisness of the eigensystem based on $p$-subordination, see e.g. [12, Thm.XIX.2.7] or [24, Thm.6.12], cannot provide satisfactory answers for (1.1).

The goal of this paper is to analyze deeper the perturbation problems, both on the abstract level and on concrete example (1.1). Our approach is based on a condition that measures the strength of the perturbation in a more subtle way than the ordinary $p$-subordination. In detail, we work in the following setting.

Let $A$ be a self-adjoint operator with compact resolvent in a separable Hilbert space $\mathcal{H}$. Let the eigenvalues $\left\{\mu_{k}\right\}_{k \in \mathbb{N}}$ of $A$ be positive, eventually simple and satisfy

$$
\begin{aligned}
& A \psi_{k}=\mu_{k} \psi_{k}, \quad\left\|\psi_{k}\right\|=1, \\
& \exists \gamma>0, \exists \kappa>0, \exists N_{0}>0, \forall k \geq N_{0}, \quad \mu_{k+1}-\mu_{k} \geq \kappa k^{\gamma-1} ;
\end{aligned}
$$

by $\left\{\psi_{k}\right\}$ we denote normalized eigenvectors of $A$ related to $\left\{\mu_{k}\right\}$. The key assumption on the form $b$, representing the perturbation, is the so-called local formsubordination condition

$$
\exists \alpha \in \mathbb{R}, 2 \alpha+\gamma>1, \quad \exists M_{b}>0, \quad \forall m, n \in \mathbb{N}, \quad\left|b\left(\psi_{m}, \psi_{n}\right)\right| \leq \frac{M_{b}}{m^{\alpha} n^{\alpha}} .
$$

The main abstract result, Theorem 3.4, states that if conditions (1.9) and (1.10) are satisfied, then the eigensystem of the perturbed operator $T$ contains a Riesz basis of $\mathcal{H}$; the operator $T$ is defined via the form sum $a+b$, where is $a$ associated with $A$. (For details on the definition of $T$, see Section 2; for the completeness of the eigensystem of $T$ in $\mathcal{H}$, see Proposition 2.1). Moreover, the eigenvalues of $T$ are eventually simple, the usual asymptotic formulas for the corrections of $\left\{\mu_{k}\right\}$ and $\left\{\psi_{k}\right\}$ hold and remainder estimates, expressed in terms of $\alpha$ and $\gamma$, are given, see Theorem 3.2 . 
The applicability of conditions (1.9) and (1.10) is demonstrated on Schrödinger operator $T$ from (1.1) viewed as a perturbation of the self-adjoint $A$ from (1.2). The condition (1.9) is satisfied for this $A$ with $\gamma=2 \beta /(\beta+2)$, see Proposition 6.1. On the other hand, for $\beta \geq 2$, the form $b$ generated by the potential $V$ satisfies the condition (1.10) if e.g. $V=V_{1}+V_{2}+V_{3}+V_{4}$ where

$$
\begin{aligned}
& \exists \varepsilon>0, \quad|x|^{\frac{2-\beta}{2}+\varepsilon} V_{1}(x) \in L^{\infty}(\mathbb{R}), \\
& \exists p \in[1, \infty), \quad V_{2} \in L^{p}(\mathbb{R}), \\
& \exists s \in\left[0, \frac{\beta-1}{2 \beta}\right), \quad V_{3} \in W^{-s, 2}(\mathbb{R}), \\
& \exists\left\{\nu_{k}\right\} \in \ell^{1}(\mathbb{Z}), \quad \exists\left\{x_{k}\right\} \subset \mathbb{R}, \quad V_{4}=\sum_{k \in \mathbb{Z}} \nu_{k} \delta\left(x-x_{k}\right),
\end{aligned}
$$

see Theorems 6.4, 6.9 and Corollaries 6.5, 6.10 for precise statements (with $\beta<2$ allowed). Moreover, for $V \in L^{1}(\mathbb{R})$ with a controlled decay at infinity, see Theorem 6.6 , we prove that the first corrections of $\mu_{k}$ read (with the precisely determined constant $C_{\beta}$ )

$$
\lambda_{n}^{(1)}=C_{\beta} n^{-\frac{2}{\beta+2}} \int_{\mathbb{R}} V(x) \mathrm{d} x+o\left(n^{-\frac{2}{\beta+2}}\right), \quad n \rightarrow \infty .
$$

Although our main motivation are Schrödinger operators $T$ from (1.1), the abstract results are applicable to other problems. In particular, see Section 5.2 for infinite finite band matrices and Section 5.3 for perturbations of $-\mathrm{d}^{2} / \mathrm{d} x^{2}$ on a finite interval with Neumann boundary conditions. For the latter, some classical results, e.g. the Riesz basisness of the eigensystem for the separated boundary conditions, see [12, Chap.XIX.3], follow immediately when formulated in our setting. The efficiency of our approach can be further illustrated on that the amount of effort needed to prove the Riesz basisness for $-\mathrm{d}^{2} / \mathrm{d} x^{2}$ from above perturbed e.g. by the infinite number of complex $\delta$-interactions (which can hardly be treated by ODE methods) is basically the same as when perturbing by a bounded potential; see Section 5.3. As $-\mathrm{d}^{2} / \mathrm{d} x^{2}$ on $(-1,1)$ with Dirichlet boundary conditions is a limit of $A$ from (1.2) with $Q(x)=|x|^{\beta}$ for $\beta \rightarrow+\infty$, it is not surprising that by taking formally $\beta=+\infty$ in the formulas, e.g. (1.12), and conditions derived for $A$ with $\beta \in(1, \infty)$, we recover those for the limit $-\mathrm{d}^{2} / \mathrm{d} x^{2}$ on $(-1,1)$, see Remark 6.7 .

Regarding the relations to previous works, the special version of condition (1.10) with $\gamma=1$ was introduced in [29]; the relation to the operator version of $(1.10)$ used in $[2,3,34,35]$ is discussed in [29] as well. Comparing to previous papers, we allow here a faster condensation of $\left\{\mu_{k}\right\}$ at infinity, namely $\mu_{k} \sim k^{\gamma}$ with $\gamma>0$ is possible, cf. [3] with the restriction $\gamma>1 / 2$. At the same time, the proof on the main abstract Theorem 3.4 on the Riesz basisness is simplified by using only the equivalent condition for a Riesz basis, see [16, Thm.VI.2.1], together with the Schur test for infinite matrices, see e.g. [33], [17, Thm.5.2]; thereby we avoid the Kato's lemma on projections [21, Thm.V.4.17a] used in [2, 3, 29]. Moreover, condition (1.10) is sharp in the sense that it cannot be weakened to $2 \alpha+\gamma=1$, see Section 5.1. As for Schrödinger operators $T$, the form-local subordination allows for treating more singular potentials (in $L^{p}$ with $1 \leq p<2$ ) than in [3] and also the distributional ones. The asymptotic formula for the first eigenvalue correction, see (1.12) or (6.74), should be compared with precise two terms asymptotics of eigenvalues in $[27,28,30]$ for the harmonic oscillator $\left(Q(x)=x^{2}\right)$ perturbed by $\delta$-interactions (instead of $L^{1}$-potentials).

The paper is organized as follows. In Section 2 we recall the definition of the perturbed operator $T$ based on the form sum. Moreover, using the classical perturbation theory, we derive basic properties of $T$, in particular the completeness 
of its eigensystem. Main abstract results, Riesz basisness, asymptotic formulas for eigenvalues and eigenvectors, are stated and proved in Section 3. In Section 4, we prove several technical lemmas used in the proofs in Section 3. Section 5 consists of several examples, showing the optimality of our assumptions and applicability of the main theorems in simpler examples. Finally, Section 6 is devoted to the analysis of the Schödinger operators (1.1).

\section{Preliminaries}

The definition of the (abstract) perturbed operator $T$ is based on representation and perturbations theorems from $[21$, Sec.VI]. Let $A$ be a self-adjoint operator satisfying (1.9) and $b$ be a form satisfying (1.10). The operator $A$ is associated with the form

$$
a[f]:=a(f, f)=\left\|A^{\frac{1}{2}} f\right\|^{2}, \quad \operatorname{Dom}(a)=\operatorname{Dom}\left(A^{\frac{1}{2}}\right),
$$

see [21, Thm.VI.2.23] for the second representation theorem. We show below that the form $t:=a+b$ is sectorial and closed. Thus, using the first representation theorem, see [21, Thm.VI.2.1], $t$ is associated with the unique m-sectorial operator $T$, which is our perturbed operator. In particular, Schrödinger operator $T$ from (1.1) with $V \in L_{\text {loc }}^{1}(\mathbb{R})$ is obtained by choosing $A$ as in (1.2) and $b$ as the form $b_{V}$ generated by $V$, see (6.68); for the distributional potentials see (6.93).

To show that $t$ is sectorial and closed, notice that when condition (1.10) is satisfied, the form $b$ is $p$-subordinated to $a$ with some $p=p(\alpha, \gamma) \in[0,1)$,

$$
\forall f \in \operatorname{Dom}(a), \quad|b[f]| \leq C(a[f])^{p}\|f\|^{2(1-p)} ;
$$

see Lemma 4.2 for details. Hence $b$ is relatively bounded with respect to $a$ with the bound 0 in particular, i.e.

$$
\forall \varepsilon>0, \exists C_{\varepsilon} \geq 0, \forall f \in \operatorname{Dom}(a), \quad|b[f]| \leq \varepsilon a[f]+C_{\varepsilon}\|f\|^{2} .
$$

Thus the form $t=a+b$ is indeed sectorial and closed and it determines uniquely the m-sectorial operator $T$ with compact resolvent, see [21, Thm.VI.3.4]. Moreover, the norm of the resolvent of $T$ decays along every ray except $\mathbb{R}^{+}$, namely,

$$
\forall \vartheta \in(0,2 \pi), \quad \lim _{t \rightarrow+\infty}\left\|\left(e^{\mathrm{i} \vartheta} t-T\right)^{-1}\right\|=0 ;
$$

the proof is based on the relative boundedness with the bound 0 and

$$
\left\|(z-T)^{-1}\right\| \leq \frac{1}{\operatorname{dist}(z, \overline{\operatorname{Num}(T)})},
$$

where $\operatorname{Num}(T)$ denotes the numerical range of $T$, see [21, Thm.V.3.2].

The operator $T$ can be also described as follows. We introduce the operators

$$
K(z) f:=\sum_{k \in \mathbb{N}}\left(z-\mu_{k}\right)^{-\frac{1}{2}}\left\langle f, \psi_{k}\right\rangle \psi_{k}, \quad z \in \rho(A),
$$

where, for $0 \neq w \in \mathbb{C}$ and $s \in \mathbb{R}$, the $s$-power of $w$ is taken as $w^{s}:=|w|^{s} e^{\text {is } \arg w}$ with $-\pi<\arg w \leq \pi$. Notice that

$$
K(z)^{2}=(z-A)^{-1}, \quad z \in \rho(A) .
$$

Then the operator $T$ reads

$$
T=A^{\frac{1}{2}}(I-B(0)) A^{\frac{1}{2}}
$$

where $B(z), z \in \rho(A)$, is the operator uniquely determined by the bounded form

$$
b\left(K(z) \cdot, K(z)^{*} \cdot\right) .
$$


In more detail, $B(z)$ is determined from the relation $\langle B(z) f, g\rangle=b\left(K(z) f,\left(K(z)^{*} g\right)\right.$ for all $f, g \in \mathcal{H}$. For all $z \in \rho(A)$, we have

$$
z-T=K(z)^{-1}(I-B(z)) K(z)^{-1}
$$

and this relation yields a suitable factorization of the resolvent of $T$, namely

$$
(z-T)^{-1}=K(z)(I-B(z))^{-1} K(z),
$$

provided $I-B(z)$ is invertible and $z \in \rho(A)$; see also [4, Lemma 1], [21, Chap.VI.3.1].

Points $z$ for which $I-B(z)$ is invertible certainly exist since we get straightforwardly from (2.3) and (2.7) that

$$
\forall \varepsilon>0, \exists \tilde{C}_{\varepsilon} \geq 0, \forall z<0, \quad\|B(z)\| \leq \varepsilon+\frac{\tilde{C}_{\varepsilon}}{|z|} .
$$

Thus $\|B(z)\|<1$ if $z<0$ and $|z|$ is sufficiently large, hence $I-B(z)$ is invertible for such $z$ 's.

The inequality (1.9) implies that, for sufficiently large $k$, we have

$$
\exists c>0, \exists k_{0} \in \mathbb{N}, \forall k>k_{0}, \quad \mu_{k} \geq c k^{\gamma},
$$

see Lemma 4.1 for details. Thus for all $z \in \rho(A), K(z)$ is in the Schatten class $\mathcal{S}_{2 r}$ with any $r$ satisfying $r \gamma>1$. The factorization $(2.11)$ and the fact that $(I-B(z))^{-1}$ is bounded for some $z<0$ implies that

$$
\forall z \in \rho(T), \forall r>\frac{1}{\gamma}, \quad(z-T)^{-1} \in \mathcal{S}_{r} .
$$

Combining (2.14), (2.4) and [12, Cor.XI.9.31], we obtain the following.

Proposition 2.1. Let conditions (1.9), (1.10) hold and let $T$ be as in (2.8). Then the eigensystem of $T$ is complete in $\mathcal{H}$.

Finally, we remark that $T^{*}$, the adjoint of $T$, is the operator associated with the adjoint form $t^{*}=a+b^{*}$, see [21, Chap.VI.1.1] and [21, Thm.VI.2.5]. We have from the definition of the adjoint form and (1.10) that

$$
\left|b^{*}\left(\psi_{m}, \psi_{n}\right)\right|=\left|\overline{b\left(\psi_{n}, \psi_{m}\right)}\right| \leq \frac{M_{b}}{m^{\alpha} n^{\alpha}} .
$$

So the analogues of results derived for $T$ under assumptions (1.9) and (1.10) are valid also for $T^{*}$, in particular, the eigensystem of $T^{*}$ is complete.

\section{Abstract perturbation Results}

3.1. Localization of eigenvalues of $T$. For $N \in \mathbb{N}$ and $h>0$, we define sets

$$
\begin{aligned}
\Pi_{0} & =\Pi_{0}(N, h):=\left\{z \in \mathbb{C}:-h<\operatorname{Re} z<\mu_{N}+\frac{\kappa}{2} N^{\gamma-1},|\operatorname{Im} z|<h\right\}, \\
\Pi_{k} & :=\left\{z \in \mathbb{C}: \mu_{k}-\frac{\kappa}{2}(k-1)^{\gamma-1}<\operatorname{Re} z<\mu_{k}+\frac{\kappa}{2} k^{\gamma-1},|\operatorname{Im} z|<\frac{\kappa}{2} k^{\gamma-1}\right\}, \\
\Pi & :=\bigcup_{k \in \mathbb{N}} \Pi_{k}, \quad \Gamma_{0}:=\partial \Pi_{0}, \quad \Gamma_{k}:=\partial \Pi_{k}, \quad k \in \mathbb{N} .
\end{aligned}
$$

Proposition 3.1. Let conditions (1.9), (1.10) hold and let $T$ be as in (2.8). Then there exist $N>N_{0}$ and $h>0$ such that

$$
\sigma(T)=\sigma_{\mathrm{p}}(T) \subset \Pi_{0}(N, h) \cup \bigcup_{k>N} \Pi_{k},
$$

where $\Pi_{k}$ are as in (3.1). Moreover, with these $N$ and h, Riesz projections

$$
S_{N}:=\frac{1}{2 \pi \mathrm{i}} \int_{\Gamma_{0}}(z-T)^{-1} \mathrm{~d} z, \quad P_{n}:=\frac{1}{2 \pi \mathrm{i}} \int_{\Gamma_{n}}(z-T)^{-1} \mathrm{~d} z, \quad n>N,
$$


are well-defined and

$$
\operatorname{rank} S_{N}=N, \quad \operatorname{rank} P_{n}=1, \quad n>N .
$$

Proof. Our first aim is to find $N$ and $h$ such that $(z-T)^{-1}$ is bounded for all $z \notin \Pi_{0}(N, h) \cup\left(\cup_{k>N} \Pi_{k}\right)$. With the help of the resolvent factorization (2.11), it suffices to prove that $\|B(z)\| \leq 1 / 2$ for all such $z$. Let $f=\sum_{j=1}^{\infty} f_{j} \psi_{j} \in \mathcal{H}$, then

$$
\begin{aligned}
\|B(z) f\|^{2} & =\sum_{k=1}^{\infty}\left|\left\langle B(z) f, \psi_{k}\right\rangle\right|^{2}=\sum_{k=1}^{\infty}\left|\sum_{j=1}^{\infty} \frac{f_{j} b\left(\psi_{j}, \psi_{k}\right)}{\left(z-\mu_{j}\right)^{\frac{1}{2}}\left(z-\mu_{k}\right)^{\frac{1}{2}}}\right|^{2} \\
& \leq M_{b}^{2} \sum_{k=1}^{\infty} \frac{1}{k^{2 \alpha}\left|\mu_{k}-z\right|}\left(\sum_{j=1}^{\infty} \frac{\left|f_{j}\right|}{j^{\alpha}\left|\mu_{j}-z\right|^{\frac{1}{2}}}\right)^{2} \\
& \leq M_{b}^{2}\left(\sum_{k=1}^{\infty} \frac{1}{k^{2 \alpha}\left|\mu_{k}-z\right|}\right)^{2}\|f\|^{2} .
\end{aligned}
$$

From Lemma 4.4, see (4.8) for the definition of $\sigma_{\omega, \gamma}$, we obtain

$$
\sup _{\substack{z \notin \Pi \\ \operatorname{Re} z \geq \mu_{n}}} \sum_{k=1}^{\infty} \frac{1}{k^{2 \alpha}\left|\mu_{k}-z\right|}=\mathcal{O}\left(\sigma_{2 \alpha, \gamma}(n)\right), \quad n \rightarrow \infty .
$$

Hence, we can indeed choose $N>N_{0}$ such that, for all $z \notin \Pi$ and $\operatorname{Re} z \geq \mu_{N}$, we have $\|B(z)\| \leq 1 / 2$.

The next step is the estimate of $\|B(z)\|$ for Re $z \leq-h_{1}$. Splitting the final sum in (3.5) as in (4.20)-(4.21), we have

$$
\|B(z)\| \leq M_{b}\left(\frac{\max \left\{N_{0}, N_{0}^{1-2 \alpha}\right\}}{h_{1}}+\sum_{k=N_{0}+1}^{\infty} \frac{1}{k^{2 \alpha}\left(\mu_{k}+h_{1}\right)}\right) .
$$

It follows from Lemma 4.1, see also (2.13), and $2 \alpha+\gamma>1$ that

$$
\sum_{k=N_{0}+1}^{\infty} \frac{1}{k^{2 \alpha} \mu_{k}}<\infty
$$

thus

$$
\lim _{h_{1} \rightarrow \infty} \sum_{k=N_{0}+1}^{\infty} \frac{1}{k^{2 \alpha}\left(\mu_{k}+h_{1}\right)}=0 .
$$

Hence there is $h_{1}>0$ such that, for all $z$ with $\operatorname{Re} z \leq-h_{1}$, we have $\|B(z)\| \leq 1 / 2$.

In the third step, we estimate $\|B(z)\|$ for $z$ with $\operatorname{Re} z \in\left[-h_{2}, \mu_{N}+\frac{\kappa}{2} N^{\gamma-1}\right]$ and $|\operatorname{Im} z| \geq h_{2}$. As in (3.7), we get the estimate

$$
\|B(z)\| \leq M_{b}\left(\frac{C}{h_{2}}+\sum_{k=2 N}^{\infty} \frac{1}{k^{2 \alpha} \sqrt{\left(\mu_{k}-\mu_{N+1}\right)^{2}+h_{2}^{2}}}\right)
$$

and conclude that

$$
\lim _{h_{2} \rightarrow \infty} \sum_{k=2 N}^{\infty} \frac{1}{k^{2 \alpha} \sqrt{\left(\mu_{k}-\mu_{N+1}\right)^{2}+h_{2}^{2}}}=0 .
$$

Thus we can choose $h_{2}>0$ such that $\|B(z)\| \leq 1 / 2$ for all $z$ with $\operatorname{Re} z \in\left[-h_{2}, \mu_{N}+\right.$ $\left.\frac{\kappa}{2} N^{\gamma-1}\right]$ and $|\operatorname{Im} z| \geq h_{2}$.

In summary, taking $h:=\max \left\{h_{1}, h_{2}\right\}$, we have that $\|B(z)\| \leq 1 / 2$ for all $z \notin$ $\Pi_{0}(N, h) \cup\left(\cup_{k>N} \Pi_{k}\right)$, thus $(3.2)$ is proved. 
The standard argument, based on [12, Lemma VII.6.7], shows that

$$
\operatorname{Tr} \frac{1}{2 \pi \mathrm{i}} \int_{\Gamma_{n}}(z-A)^{-\frac{1}{2}}(I-t B(z))^{-1}(z-A)^{-\frac{1}{2}} \mathrm{~d} z, \quad 0 \leq t \leq 1,
$$

is a continuous integer-valued function, thus it is constant and (3.4) follows.

3.2. Asymptotics of eigenvalues and eigenvectors of $T$. It follows from Proposition 3.1 that the eigenvalues $\left\{\lambda_{n}\right\}$ of $T$ become eventually simple (for $n>N$ ) and localized around those of the unperturbed operator $A$. The rest of the spectrum is localized in $\Pi_{0}$. Therefore for $n>N$, we have $\operatorname{Tr} P_{n}=1$, see (3.3), thus

$$
\begin{aligned}
\lambda_{n}-\mu_{n} & =\frac{1}{2 \pi \mathrm{i}} \operatorname{Tr} \int_{\Gamma_{n}} z\left((z-T)^{-1}-(z-A)^{-1}\right) \mathrm{d} z \\
& =\frac{1}{2 \pi \mathrm{i}} \operatorname{Tr} \int_{\Gamma_{n}}\left(z-\mu_{n}\right)\left((z-T)^{-1}-(z-A)^{-1}\right) \mathrm{d} z, \quad n>N .
\end{aligned}
$$

As in e.g. $[13,14,26,27,28,30]$, the further analysis of $\lambda_{n}$ with $n>N$ relies on the formula (3.12). The eigenvectors $\left\{\phi_{n}\right\}_{n>N}$ of $T$, satisfying $T \phi_{n}=\lambda_{n} \phi_{n}$, are found using

$$
\begin{aligned}
\phi_{n} & =\frac{1}{2 \pi \mathrm{i}} \int_{\Gamma_{n}}(z-T)^{-1} \psi_{n} \mathrm{~d} z \\
& =\psi_{n}+\frac{1}{2 \pi \mathrm{i}} \int_{\Gamma_{n}}\left((z-T)^{-1}-(z-A)^{-1}\right) \psi_{n} \mathrm{~d} z, \quad n>N .
\end{aligned}
$$

Theorem 3.2. Let conditions (1.9), (1.10) hold and let $T$ be as in (2.8). Denote by $\left\{\lambda_{n}\right\}_{n \in \mathbb{N}}$ the eigenvalues of $T$ so that $\lambda_{n} \in \Pi_{n}$ for $n>N$. Then

$$
\lambda_{n}=\mu_{n}+\sum_{k=1}^{j} \lambda_{n}^{(k)}+r_{n}^{(j)}, \quad j \in \mathbb{N}, \quad n>N,
$$

where

$$
\lambda_{n}^{(k)}=\frac{1}{2 \pi \mathrm{i}} \operatorname{Tr} \int_{\Gamma_{n}}\left(z-\mu_{n}\right) K(z) B(z)^{k} K(z) \mathrm{d} z, \quad k \geq 1,
$$

and

$$
\left|\lambda_{n}^{(k)}\right|=\mathcal{O}\left(n^{\gamma-1} \sigma_{2 \alpha, \gamma}(n)^{k}\right), \quad\left|r_{n}^{(j)}\right|=\mathcal{O}\left(\frac{\sigma_{2 \alpha, \gamma}(n)^{j+1}}{n^{2 \alpha}}\right), \quad n \rightarrow \infty .
$$

Moreover, the vectors

$$
\phi_{n}:=\frac{1}{2 \pi \mathrm{i}} \int_{\Gamma_{n}}(z-T)^{-1} \psi_{n} \mathrm{~d} z=\psi_{n}+\sum_{k=1}^{j} \phi_{n}^{(k)}+\rho_{n}^{(j)}, \quad j \in \mathbb{N}, \quad n>N,
$$

where

$$
\phi_{n}^{(k)}=\frac{1}{2 \pi \mathrm{i}} \int_{\Gamma_{n}} K(z) B(z)^{k} K(z) \psi_{n} \mathrm{~d} z
$$

and

$$
\left\|\phi_{n}^{(k)}\right\|=\mathcal{O}\left(\sigma_{2 \alpha, \gamma}(n)^{k}\right), \quad\left\|\rho_{n}^{(j)}\right\|=\mathcal{O}\left(\sigma_{2 \alpha, \gamma}(n)^{j+1}\right), \quad n \rightarrow \infty,
$$

satisfy (with some $N_{1} \geq N$ )

$$
T \phi_{n}=\lambda_{n} \phi_{n}, \quad \phi_{n} \neq 0, \quad n>N_{1} \geq N .
$$

Proof. If $n>N$ and $z \in \Gamma_{n}$, we have $\|B(z)\|<1$, see Proposition 3.1 and its proof. Thus we can expand $(I-B(z))^{-1}$ into a convergent sum and thereby get

$$
(z-T)^{-1}-(z-A)^{-1}=K(z)\left(\sum_{m=1}^{\infty} B(z)^{m}\right) K(z) .
$$


Inserting (3.21) into (3.12) and (3.13), we obtain (3.14), (3.15) and (3.17), (3.18). The claim (3.20) follows from (3.17) and (3.19).

The main part of the proof is the explanation that the operators in (3.15) are indeed trace class and that the estimates (3.16), (3.19) hold. In what follows, we denote the $r$-Schatten class norm by $\|\cdot\|_{r}$. The estimates are done mostly in the same way as in [27, Sec.2.4].

We start with $K(z) B(z) K(z)$, so $k=1$ in (3.15) and (3.16). Using

$$
B(z) \psi_{j}=\sum_{m=1}^{\infty}\left\langle B(z) \psi_{j}, \psi_{m}\right\rangle \psi_{m}
$$

we obtain

$$
K(z) B(z) K(z) \psi_{j}=\sum_{m=1}^{\infty} \frac{b\left(\psi_{j}, \psi_{m}\right)}{\left(z-\mu_{j}\right)\left(z-\mu_{m}\right)} \psi_{m}, \quad z \in \Gamma_{n} .
$$

The integration leads to

$$
\begin{aligned}
& \frac{1}{2 \pi \mathrm{i}} \int_{\Gamma_{n}}\left(z-\mu_{n}\right)\left\langle K(z) B(z) K(z) \psi_{j}, \psi_{l}\right\rangle \mathrm{d} z \\
& \quad=\frac{1}{2 \pi \mathrm{i}} \int_{\Gamma_{n}} \frac{\left(z-\mu_{n}\right) b\left(\psi_{j}, \psi_{l}\right)}{\left(z-\mu_{j}\right)\left(z-\mu_{l}\right)} \mathrm{d} z=b\left(\psi_{n}, \psi_{n}\right) \delta_{j, n} \delta_{l, n}
\end{aligned}
$$

Thus the operator in (3.15) reads

$$
\frac{1}{2 \pi \mathrm{i}} \int_{\Gamma_{n}}\left(z-\mu_{n}\right) K(z) B(z) K(z) \mathrm{d} z=b\left(\psi_{n}, \psi_{n}\right)\left\langle\cdot, \psi_{n}\right\rangle,
$$

and so it is of rank one and hence trace class. Moreover, $\left|\lambda_{n}^{(1)}\right|=\mathcal{O}\left(n^{-2 \alpha}\right), n \rightarrow \infty$, so (3.16) holds for $k=1$.

Next we show that $B(z) \in \mathcal{S}_{2}$ for all $z \in \Gamma_{n}, n>N$, and

$$
\sup _{z \in \Gamma_{n}}\|B(z)\|_{2}=\mathcal{O}\left(\sigma_{2 \alpha, \gamma}(n)\right), \quad n \rightarrow \infty .
$$

Indeed, using again (3.22), for $z \in \Gamma_{n}, n>N$, we obtain

$$
\begin{aligned}
\|B(z)\|_{2}^{2} & =\sum_{m=1}^{\infty}\left|\left\langle B(z) \psi_{m}, B(z) \psi_{m}\right\rangle\right|^{2}=\sum_{j, m=1}^{\infty} \frac{\left|b\left(\psi_{m}, \psi_{j}\right)\right|^{2}}{\left|z-\mu_{j}\right|\left|z-\mu_{m}\right|} \\
& \leq M_{b}^{2}\left(\sum_{m=1}^{\infty} \frac{1}{m^{2 \alpha}\left|z-\mu_{m}\right|}\right)^{2}=\mathcal{O}\left(\sigma_{2 \alpha, \gamma}(n)^{2}\right), \quad n \rightarrow \infty
\end{aligned}
$$

in the last step, we use Lemma 4.4. Hence we have $K(z) B(z)^{k} K(z) \in \mathcal{S}_{1}$ for $k \geq 2$ and all $z \in \Gamma_{n}$ with $n>N$. From $|\operatorname{Tr} C| \leq\|C\|_{1}$, see [12, Cor.XI.9.8], $\|\bar{K}(z)\|^{2}=1 /\left|z-\mu_{n}\right|$ if $z \in \Gamma_{n},(3.27),\left|\Gamma_{n}\right|=\overline{\mathcal{O}}\left(n^{\gamma-1}\right)$ as $n \rightarrow \infty$ and [12, Lemma XI.9.14], we have

$$
\begin{aligned}
\left|\lambda_{n}^{(k)}\right| & \leq \frac{1}{2 \pi} \int_{\Gamma_{n}}\left|z-\mu_{n}\right|\|K(z)\|^{2}\|B(z)\|_{2}^{k}|\mathrm{~d} z| \\
& =\mathcal{O}\left(n^{\gamma-1} \sigma_{2 \alpha, \gamma}(n)^{k}\right), \quad n \rightarrow \infty .
\end{aligned}
$$

Moreover, for the remainder $r_{n}^{(j)}, j \geq 2$, we have

$$
\begin{aligned}
\left|r_{n}^{(j)}\right| & \leq \sum_{m=j+1}^{\infty} \frac{1}{2 \pi} \int_{\Gamma_{n}}\left|z-\mu_{n}\right|\|K(z)\|^{2}\|B(z)\|_{2}^{m}|\mathrm{~d} z| \\
& =\mathcal{O}\left(n^{\gamma-1} \sigma_{2 \alpha, \gamma}(n)^{j+1}\right), \quad n \rightarrow \infty,
\end{aligned}
$$


thus (3.16) is proved. Finally for the eigenvectors, we get from (3.18), (3.27) that

$$
\begin{aligned}
\left\|\phi_{n}^{(k)}\right\| & \leq \frac{1}{2 \pi} \int_{\Gamma_{n}}\|K(z)\|^{2}\|B(z)\|_{2}^{k}|\mathrm{~d} z|=\mathcal{O}\left(\sigma_{2 \alpha, \gamma}(n)^{k}\right), \quad n \rightarrow \infty \\
\left\|\rho_{n}^{(j)}\right\| & \leq \sum_{m=j+1}^{\infty} \frac{1}{2 \pi} \int_{\Gamma_{n}}\|K(z)\|^{2}\|B(z)\|_{2}^{m}|\mathrm{~d} z|=\mathcal{O}\left(\sigma_{2 \alpha, \gamma}(n)^{j+1}\right), \quad n \rightarrow \infty
\end{aligned}
$$

so (3.19) is proved as well.

Remark 3.3. In particular, we have

$$
\begin{aligned}
& \lambda_{n}^{(1)}=b\left(\psi_{n}, \psi_{n}\right), \quad \lambda_{n}^{(2)}=\sum_{j=1, j \neq n}^{\infty} \frac{\left|b\left(\psi_{n}, \psi_{j}\right)\right|^{2}}{\mu_{n}-\mu_{j}} \\
& \phi_{n}^{(1)}=\sum_{j=1, j \neq n}^{\infty} \frac{b\left(\psi_{n}, \psi_{j}\right)}{\mu_{n}-\mu_{j}} \psi_{j} .
\end{aligned}
$$

Proof. Formulas for $\lambda_{n}^{(2)}$ and $\phi_{n}^{(1)}$ follow from (3.15) and (3.18), respectively, by the calculation of residues. To derive the formula for $\lambda_{n}^{(1)}$, we can use (3.25); like e.g. in [11, Lemmas 8, 9], it is important to integrate before taking the trace (or norm in the proof of Theorem 3.2) since all but one term in (3.24) are zero after the integration.

3.3. Riesz basis property of the eigensystem. Proposition 3.1 shows that there are only finitely many eigenvalues of $T$ in $\Pi_{0}$, namely $\left\{\lambda_{n}\right\}_{n=1}^{N^{\prime}}, N^{\prime} \leq N$, with algebraic multiplicities $\left\{m_{n}\right\}_{n=1}^{N^{\prime}}, \sum_{n=1}^{N^{\prime}} m_{n}=N$. The remaining eigenvalues $\left\{\lambda_{n}\right\}_{n>N}$ are simple. Hence the eigensystem of $T$ contains at most a finite number of root vectors associated with $\left\{\lambda_{n}\right\}_{n=1}^{N^{\prime}}$ and the rest consists of eigenvectors related to $\left\{\lambda_{n}\right\}_{n>N}$. For $n>N_{1}$, these eigenvectors can be selected as $\left\{\phi_{n}\right\}_{n>N_{1}}$ from Theorem 3.2.

Theorem 3.4. Let $A$ be a self-adjoint operator with compact resolvent in a Hilbert space $\mathcal{H}$, let (1.9) hold and let a be the sesquilinear form associated with $A$. Let $b$ be a sesquilinear form satisfying (2.8) and let $T$ be the operator associated with the form $t=a+b$. Let $N_{1}>N$ be such that (3.20) holds for all $n>N_{1}$ and let $S_{N_{1}}$ be the spectral projection of $T$ from (3.3) with $N=N_{1}$.

Then the set $\left\{\phi_{n}\right\}_{n=1}^{\infty}$, where $\left\{\phi_{n}\right\}_{n=1}^{N_{1}}$ is a basis of $\operatorname{Ran}\left(S_{N_{1}}\right)$ and $\phi_{n}$ with $n>N_{1}$ are eigenvectors of $T$ from (3.17) related to simple eigenvalues $\lambda_{n}, n>N_{1}$, is a Riesz basis of $\mathcal{H}$.

Proof. The proof is based on [16, Thm.VI.2.1] and Schur test for infinite matrices, see e.g. [33], [17, Thm.5.2]. We need to verify that $\left\{\phi_{n}\right\}$ is complete in $\mathcal{H}$, there exists a complete system $\left\{\tilde{\phi}_{n}\right\}$ that is biorthogonal to $\left\{\phi_{n}\right\}$ and we have

$$
\forall f \in \mathcal{H}, \quad \sum_{n=1}^{\infty}\left|\left\langle f, \phi_{n}\right\rangle\right|^{2}<\infty, \quad \sum_{n=1}^{\infty}\left|\left\langle f, \tilde{\phi}_{n}\right\rangle\right|^{2}<\infty .
$$

The system $\left\{\phi_{n}\right\}$ is complete by Proposition 2.1. As the biorthogonal system, we can select vectors $\tilde{\phi}_{n}$ from the eigensystem of $T^{*}$ with

$$
\tilde{\phi}_{n}:=\frac{1}{\left\langle\phi_{n}^{*}, \phi_{n}\right\rangle} \phi_{n}^{*}, \quad \phi_{n}^{*}:=\frac{1}{2 \pi \mathrm{i}} \int_{\Gamma_{n}}\left(z-T^{*}\right)^{-1} \psi_{n} \mathrm{~d} z, \quad n>N_{1} .
$$

Due to (2.15), we obtain as in Theorem 3.2 that

$$
\phi_{n}^{*}=\psi_{n}+\sum_{k=1}^{j} \phi_{n}^{*(k)}+\rho_{n}^{*(j)}, \quad j \in \mathbb{N}
$$


where

$$
\phi_{n}^{*(k)}=\frac{1}{2 \pi \mathrm{i}} \int_{\Gamma_{n}} K(z)\left(B(\bar{z})^{*}\right)^{k} K(z) \psi_{n} \mathrm{~d} z
$$

and

$$
\left\|\phi_{n}^{*(k)}\right\|=\mathcal{O}\left(\sigma_{2 \alpha, \gamma}(n)^{k}\right), \quad\left\|\rho_{n}^{*(j)}\right\|=\mathcal{O}\left(\sigma_{2 \alpha, \gamma}(n)^{j+1}\right), \quad n \rightarrow \infty ;
$$

notice that then $\left\langle\phi_{n}^{*}, \phi_{n}\right\rangle=1+\mathcal{O}\left(\sigma_{2 \alpha, \gamma}(n)\right)$ as $n \rightarrow \infty$. Moreover, the system $\left\{\tilde{\phi}_{n}\right\}$ is complete in $\mathcal{H}$; see remarks below Proposition 2.1.

The crucial step is to show (3.32). We analyze the sum with $\left\{\phi_{n}\right\}$ only. In view of (2.15) and (3.34)-(3.36), the reasoning for the second sum is completely analogous. Clearly, it suffices to consider the sum for $n>N_{1}$ only. We give the detailed proof for the case $2 \alpha \leq 1$, the other case is similar.

First select $j \in \mathbb{N}$ such that $2(j+1)(2 \alpha+\gamma-1)>1$, then, using (3.19) and (4.8), we get

$$
\sum_{n=N_{1}+1}^{\infty}\left|\left\langle f, \rho_{n}^{(j)}\right\rangle\right|^{2} \leq\|f\|^{2} \sum_{n=N_{1}+1}^{\infty}\left\|\rho_{n}^{(j)}\right\|^{2}<\infty .
$$

Thus it remains to estimate $\left|\left\langle f, \phi_{n}^{(k)}\right\rangle\right|^{2}$ for $k=1, \ldots, j$. We first derive that

$$
B(z)^{k} \psi_{j_{0}}=\sum_{j_{1}, \ldots, j_{k}}\left(\prod_{l=1}^{k}\left\langle B(z) \psi_{j_{l-1}}, \psi_{j_{l}}\right\rangle\right) \psi_{j_{k}}
$$

and hence from (3.18)

$$
\phi_{n}^{(k)}=\frac{1}{2 \pi \mathrm{i}} \int_{\Gamma_{n}} \sum_{j_{1}, \ldots, j_{k}} \frac{b\left(\psi_{n}, \psi_{j_{1}}\right) \prod_{l=2}^{k} b\left(\psi_{j_{l-1}}, \psi_{j_{l}}\right)}{\left(z-\mu_{n}\right) \prod_{l=1}^{k}\left(z-\mu_{j_{l}}\right)} \psi_{j_{k}} \mathrm{~d} z
$$

Decomposing $f=\sum_{m=1}^{\infty} f_{m} \psi_{m}$, we obtain from (3.39) and (1.10) that

$$
\begin{aligned}
\left|\left\langle f, \phi_{n}^{(k)}\right\rangle\right| \leq & M_{b}^{k} \frac{1}{2 \pi} \int_{\Gamma_{n}} \sum_{j_{1}, \ldots, j_{k}} \frac{\left|f_{j_{k}}\right||\mathrm{d} z|}{\left|z-\mu_{n}\right|\left|z-\mu_{j_{k}}\right| n^{\alpha} j_{k}^{\alpha} \prod_{l=1}^{k-1}\left|z-\mu_{j_{l}}\right| j_{l}^{2 \alpha}} \\
\leq & M_{b}^{k}\left(\sum_{j=1}^{\infty} \frac{1}{j^{2 \alpha}\left|z_{n}-\mu_{j}\right|}\right)^{k-1} \sum_{m=1}^{\infty} \frac{\left|f_{m}\right|}{\left|z_{n}-\mu_{m}\right| m^{\alpha} n^{\alpha}} \times \\
& \frac{1}{2 \pi} \int_{\Gamma_{n}} \frac{|\mathrm{d} z|}{\left|z_{n}-\mu_{n}\right|},
\end{aligned}
$$

where $z_{n} \in \Gamma_{n}$ is such that the maximum of the integrand in the first integral in (3.40) is attained; notice that $z_{n}$ depends on $f$. From Lemma 4.3 and $\left|\Gamma_{n}\right|=$ $\mathcal{O}\left(n^{\gamma-1}\right)$ we get further that there is a constant $C>0$ such that

$$
\left|\left\langle f, \phi_{n}^{(k)}\right\rangle\right|^{2} \leq C \sigma_{2 \alpha, \gamma}(n)^{2(k-1)}\left(\sum_{m=1}^{\infty} \frac{\left|f_{m}\right|}{\left|z_{n}-\mu_{m}\right| m^{\alpha} n^{\alpha}}\right)^{2} .
$$

The final step is to estimate the sum of $\left|\left\langle f, \phi_{n}^{(k)}\right\rangle\right|^{2}$ for $n>N_{1}$. Since $2 \alpha+\gamma-1>0$, see also (4.8), it suffices to consider the case $k=1$ only. For the latter, we get

$$
\sum_{n=N_{1}+1}^{\infty}\left|\left\langle f, \phi_{n}^{(1)}\right\rangle\right|^{2} \leq C \sum_{n=1}^{\infty}\left(\sum_{m=1}^{\infty} \frac{\left|f_{m}\right|}{m^{\alpha} n^{\alpha}\left|z_{n}-\mu_{m}\right|}\right)^{2}=C\|\mathcal{M} \tilde{f}\|_{\ell^{2}(\mathbb{N})}^{2}
$$

where $\mathcal{M}$ is an operator acting in $\ell^{2}(\mathbb{N})$ with matrix elements

$$
\mathcal{M}_{m n}=\frac{1}{m^{\alpha} n^{\alpha}\left|z_{n}-\mu_{m}\right|}, \quad m, n \in \mathbb{N}
$$


and $\tilde{f}=\left\{\left|f_{m}\right|\right\} \in \ell^{2}(\mathbb{N})$. To estimate $\|\mathcal{M}\|$ we employ the Schur test. By applying Lemma 4.4 and its slight modification for the second sum, we get

$$
\begin{gathered}
\sum_{m=1}^{\infty}\left|\mathcal{M}_{m n}\right| \frac{1}{m^{\alpha}}=\sum_{m=1}^{\infty} \frac{1}{n^{\alpha} m^{2 \alpha}\left|z_{n}-\mu_{m}\right|} \leq \frac{C_{1}}{n^{\alpha}} \frac{\log e n}{n^{2 \alpha+\gamma-1}} \leq \frac{C_{2}}{n^{\alpha}} \\
\sum_{n=1}^{\infty}\left|\mathcal{M}_{m n}\right| \frac{1}{n^{\alpha}}=\sum_{n=1}^{\infty} \frac{1}{n^{2 \alpha} m^{\alpha}\left|z_{n}-\mu_{m}\right|} \leq \frac{C_{3}}{m^{\alpha}} \frac{\log e m}{m^{2 \alpha+\gamma-1}} \leq \frac{C_{4}}{m^{\alpha}}
\end{gathered}
$$

thus the Schur test yields that $\|\mathcal{M}\|<\infty$. The latter, (3.42), (3.41) and (3.37) show that (3.32) holds for $\left\{\phi_{n}\right\}$.

Remark 3.5. Let conditions (1.9), (1.10) hold and let, in addition,

$$
\left\{\begin{array}{lll}
2 \alpha+\gamma>\frac{3}{2} & \text { if } & \alpha \leq \frac{1}{2} \\
\gamma>\frac{1}{2} & \text { if } & \alpha>\frac{1}{2} .
\end{array}\right.
$$

Then the system $\left\{\phi_{n}\right\}_{n=1}^{\infty}$ from Theorem 3.4 is a Bari basis, namely

$$
\sum_{n=1}^{\infty}\left\|\psi_{n}-\phi_{n}\right\|^{2}<\infty
$$

Proof. Inserting (3.17) into (3.46), we infer that, for $n>N_{1}$,

$$
\left\|\psi_{n}-\phi_{n}\right\|^{2} \leq 2\left(\left\|\phi_{n}^{(1)}\right\|^{2}+\left\|\rho_{n}^{(1)}\right\|^{2}\right)=\mathcal{O}\left(\sigma_{2 \alpha, \gamma}(n)^{2}\right), \quad n \rightarrow \infty .
$$

Thus conditions (3.45) imply that (3.46) holds by (4.8).

\section{TEChNiCAL LEMmas}

Lemma 4.1. Let $\left\{\mu_{k}\right\}_{k \in \mathbb{N}}$ satisfy (1.9) and let $j, k \in \mathbb{N}, k>j>N_{0}$. Then

$$
\mu_{k}-\mu_{j} \geq \frac{\kappa}{\gamma} \begin{cases}(k-1)^{\gamma}-(j-1)^{\gamma}, & \gamma \geq 1, \\ k^{\gamma}-j^{\gamma}, & 0<\gamma<1 .\end{cases}
$$

Thus,

$$
\mu_{k}-\mu_{j} \geq \frac{\kappa}{\gamma}\left((k-1)^{\gamma}-j^{\gamma}\right), \quad \gamma>0
$$

Proof. From (1.9), we get

$$
\begin{aligned}
\mu_{k}-\mu_{j} & =\sum_{i=j+1}^{k}\left(\mu_{i}-\mu_{i-1}\right) \geq \kappa \sum_{i=j+1}^{k}(i-1)^{\gamma-1} \\
& \geq \kappa \begin{cases}\int_{j}^{k}(x-1)^{\gamma-1} \mathrm{~d} x, & \gamma \geq 1 \\
\int_{j}^{k} x^{\gamma-1} \mathrm{~d} x, & 0<\gamma<1 .\end{cases}
\end{aligned}
$$

The proof is concluded by the direct integration and simple manipulations.

Lemma 4.2. Let $A$ and $b$ satisfy (1.9) and (1.10). Then for every $\tau \in(0,2 \alpha+$ $\gamma-1)$, there exists $C>0$ such that, with $p=\max \{0,1-\tau / \gamma\}$,

$$
\forall f \in \operatorname{Dom}(a), \quad|b[f]| \leq C(a[f])^{p}\|f\|^{2(1-p)} .
$$

Proof. We write $f=\sum_{j=1}^{\infty} f_{j} \psi_{j}$. Using Lemma 4.1, see also (2.13), we can verify that the following lower bound for $a[f]$ holds with some $C_{1}>0$

$$
a[f]=\sum_{j=1}^{\infty} \mu_{j}\left|f_{j}\right|^{2} \geq C_{1} \sum_{j=1}^{\infty} j^{\gamma}\left|f_{j}\right|^{2} .
$$


Using condition (1.10) and Hölder inequality, we get (with $2(\alpha+\beta)>1, \gamma>2 \beta$ )

$$
\begin{aligned}
|b[f]| & =\left|\sum_{j, k=1}^{\infty} f_{j} \bar{f}_{k} b\left(\psi_{j}, \psi_{k}\right)\right| \leq M_{b}\left(\sum_{j=1}^{\infty} \frac{\left|f_{j}\right|}{j^{\alpha}}\right)^{2}=M_{b}\left(\sum_{j=1}^{\infty}\left|f_{j}\right| j^{\beta} \frac{1}{j^{\alpha+\beta}}\right)^{2} \\
& \leq M_{b}\left(\sum_{j=1}^{\infty} j^{2 \beta}\left|f_{j}\right|^{2}\right) \sum_{j=1}^{\infty} \frac{1}{j^{2(\alpha+\beta)}} \leq C_{2}\left(\sum_{j=1}^{\infty} j^{\gamma}\left|f_{j}\right|^{2}\right)^{\frac{2 \beta}{\gamma}}\|f\|^{2\left(1-\frac{2 \beta}{\gamma}\right)} .
\end{aligned}
$$

The inequality in (4.4) follows by combining (4.5) and (4.6) and putting $2 \beta:=\gamma-\tau$ when $\alpha \leq 1 / 2$ and $\beta:=0$ when $\alpha>1 / 2$.

Lemma 4.3. Let $n \in \mathbb{N}, \gamma>0$ and $\omega+\gamma>1$. Then

$$
\sum_{k=1, k \neq n}^{\infty} \frac{1}{k^{\omega}\left|k^{\gamma}-n^{\gamma}\right|}=\mathcal{O}\left(\sigma_{\omega, \gamma}(n)\right)
$$

where

$$
\sigma_{\omega, \gamma}(n):= \begin{cases}n^{-\omega-\gamma+1} \log e n, & \omega \leq 1 \\ n^{-\gamma}, & \omega>1\end{cases}
$$

Proof. The absolute constant is denoted by the letter $C$ and can vary from line to line. In all estimates below, we assume that $n \in \mathbb{N}$ is sufficiently large. Clearly,

$$
\sum_{\substack{k=1 \\ k \neq n}}^{\infty} \frac{1}{k^{\omega}\left|k^{\gamma}-n^{\gamma}\right|} \leq \sum_{k=1}^{n-1} \frac{1}{k^{\omega}\left(n^{\gamma}-k^{\gamma}\right)}+\sum_{k=n+2}^{\infty} \frac{1}{k^{\omega}\left(k^{\gamma}-n^{\gamma}\right)}+\mathcal{O}\left(n^{-\omega-\gamma+1}\right) .
$$

The first term on the right of (4.9) can be estimated as

$$
\begin{aligned}
\sum_{k=1}^{n-1} \frac{1}{k^{\omega}\left(n^{\gamma}-k^{\gamma}\right)} & =\left(\sum_{k=1}^{\left[\frac{n}{2}\right]}+\sum_{k=\left[\frac{n}{2}\right]}^{n-1}\right) \frac{1}{k^{\omega}\left(n^{\gamma}-k^{\gamma}\right)} \\
& \leq C\left(\frac{1}{n^{\gamma}} \sum_{k=1}^{n} \frac{1}{k^{\omega}}+\frac{1}{n^{\omega}} \sum_{k=\left[\frac{n}{2}\right]}^{n-1} \frac{1}{n^{\gamma}-k^{\gamma}}\right)
\end{aligned}
$$

and similarly the second term of (4.9) as

$$
\begin{aligned}
\sum_{k=n+2}^{\infty} \frac{1}{k^{\omega}\left(k^{\gamma}-n^{\gamma}\right)} & =\left(\sum_{k=n+2}^{2 n}+\sum_{k=2 n+1}^{\infty}\right) \frac{1}{k^{\omega}\left(k^{\gamma}-n^{\gamma}\right)} \\
& \leq C\left(\frac{1}{n^{\omega}} \sum_{k=n+2}^{2 n} \frac{1}{k^{\gamma}-n^{\gamma}}+\sum_{k=2 n+1}^{\infty} \frac{1}{k^{\omega+\gamma}}\right)
\end{aligned}
$$

For a monotone, continuous, non-negative function $f$ in interval $[a, b], a, b \in \mathbb{N}$, $a<b$, we have

$$
\sum_{i=a}^{b} f(i) \leq f(a)+f(b)+\int_{a}^{b} f(x) \mathrm{d} x
$$


$f(a)$ can be omitted if $f$ is increasing and similarly $f(b)$ can be omitted if $f$ is decreasing. Thus applying (4.12), we get

$$
\begin{array}{r}
\sum_{k=1}^{n} \frac{1}{k^{\omega}} \leq 1+\frac{1}{n^{\omega}}+\int_{1}^{n} \frac{\mathrm{d} x}{x^{\omega}} \leq C \begin{cases}n^{1-\omega}, & \omega<1, \\
\log n, & \omega=1, \\
1, & \omega>1,\end{cases} \\
\sum_{k=2 n+1}^{\infty} \frac{1}{k^{\omega+\gamma}} \leq \frac{1}{(2 n+1)^{\omega+\gamma}}+\int_{2 n+1}^{\infty} \frac{\mathrm{d} x}{x^{\omega+\gamma}} \leq \frac{C}{n^{\omega+\gamma-1}} .
\end{array}
$$

Moreover, since $(1-y) /\left(1-y^{\gamma}\right) \rightarrow 1 / \gamma$ as $y \rightarrow 1$, we obtain

$$
\begin{aligned}
\sum_{k=\left[\frac{n}{2}\right]}^{n-1} \frac{1}{n^{\gamma}-k^{\gamma}} & \leq \frac{1}{n^{\gamma}-(n-1)^{\gamma}}+\int_{\frac{n}{2}-1}^{n-1} \frac{\mathrm{d} x}{n^{\gamma}-x^{\gamma}} \\
& \leq \frac{C}{n^{\gamma-1}}\left(1+\int_{\frac{1}{2}-\frac{1}{n}}^{1-\frac{1}{n}} \frac{\mathrm{d} y}{1-y^{\gamma}}\right) \leq C \frac{\log n}{n^{\gamma-1}} \\
\sum_{k=n+2}^{2 n} \frac{1}{k^{\gamma}-n^{\gamma}} & \leq \frac{C}{n^{\gamma-1}}\left(1+\int_{1+\frac{2}{n}}^{2} \frac{\mathrm{d} y}{y^{\gamma}-1}\right) \leq C \frac{\log n}{n^{\gamma-1}}
\end{aligned}
$$

Combing all the inequalities above, we receive (4.7).

Lemma 4.4. Let conditions (1.9) and $\omega+\gamma>1$ hold. Then

$$
\sup _{\substack{z \notin \Pi \\ \operatorname{Re} z \geq \mu_{n}}} \sum_{k=1}^{\infty} \frac{1}{k^{\omega}\left|\mu_{k}-z\right|}=\mathcal{O}\left(\sigma_{\omega, \gamma}(n)\right), \quad n \rightarrow \infty
$$

where $\sigma_{\omega, \gamma}(n)$ is as in (4.8) and $\Pi$ as in (3.1).

Proof. Define sets in $\mathbb{C}$

$$
\Xi_{j}:=\left\{z \notin \Pi: \operatorname{Re} z \in\left[\mu_{j-1}+\frac{\kappa}{2}(j-1)^{\gamma-1}, \mu_{j+1}-\frac{\kappa}{2} j^{\gamma-1}\right]\right\}, \quad j \in \mathbb{N}
$$

note that we can cover the region $\left\{z \notin \Pi: \operatorname{Re} z \geq \mu_{n}\right\}$ by $\cup_{j \geq n} \Xi_{j}$. In all estimates below, we assume that $n$ is sufficiently large, in particular $n>N_{0}+3$.

As we do not have information on $\left\{\mu_{k}\right\}_{k=1}^{N_{0}}$, we split the sum in (4.18),

$$
\sum_{k=1}^{\infty} \frac{1}{k^{\omega}\left|\mu_{k}-z\right|}=\left(\sum_{k=1}^{N_{0}}+\sum_{k=N_{0}+1}^{\infty}\right) \frac{1}{k^{\omega}\left|\mu_{k}-z\right|}
$$

and do a rough estimate of the first finite sum. Namely, using Lemma 4.1 in the last step, we get

$$
\sup _{\substack{z \notin \Pi \\ \operatorname{Re} z \geq \mu_{n}}} \sum_{k=1}^{N_{0}} \frac{1}{k^{\omega}\left|\mu_{k}-z\right|} \leq \frac{\max \left\{N_{0}, N_{0}^{1-\omega}\right\}}{\mu_{n}-\mu_{N_{0}}}=\mathcal{O}\left(n^{-\gamma}\right), \quad n \rightarrow \infty
$$


The second sum in (4.20) is estimated with the help of Lemmas 4.1 and 4.3,

$$
\begin{aligned}
& \sup _{\substack{z \notin \Pi \\
\operatorname{Re} z \geq \mu_{n}}} \sum_{k=N_{0}+1}^{\infty} \frac{1}{k^{\omega}\left|\mu_{k}-z\right|} \leq \sup _{j \geq n} \sup _{z_{j} \in \Xi_{j}} \sum_{k=N_{0}+1}^{\infty} \frac{1}{k^{\omega}\left|\mu_{k}-z_{j}\right|} \\
& \leq \sup _{j \geq n}\left(\sum_{k=N_{0}+1}^{j-3} \frac{1}{k^{\omega}\left(\mu_{j-1}-\mu_{k}\right)}+\sum_{k=j+3}^{\infty} \frac{1}{k^{\omega}\left(\mu_{k}-\mu_{j+1}\right)}\right) \\
& \quad+\sup _{j \geq n} \sup _{z_{j} \in \Xi_{j}} \sum_{k=j-2}^{j+3} \frac{1}{k^{\omega}\left|\mu_{k}-z_{j}\right|} .
\end{aligned}
$$

From the definition of sets $\Xi_{j}$, see (4.19), the last term on the right of $(4.22)$ is $\mathcal{O}\left(n^{1-\omega-\gamma}\right)$ as $n \rightarrow \infty$. The remaining terms in (4.22) are estimated using Lemma 4.1 in the first step and Lemma 4.3 in the second step

$$
\begin{aligned}
& \sup _{j \geq n}\left(\sum_{k=N_{0}+1}^{j-3} \frac{1}{k^{\omega}\left(\mu_{j-1}-\mu_{k}\right)}+\sum_{k=j+3}^{\infty} \frac{1}{k^{\omega}\left(\mu_{k}-\mu_{j+1}\right)}\right) \\
& \leq \frac{\kappa}{\gamma} \sup _{j \geq n}\left(\sum_{k=N_{0}+1}^{j-3} \frac{1}{k^{\omega}\left((j-2)^{\gamma}-k^{\gamma}\right)}+\sum_{k=j+3}^{\infty} \frac{1}{k^{\omega}\left((k-1)^{\gamma}-(j+1)^{\gamma}\right)}\right) \\
& \quad=\mathcal{O}\left(\sigma_{\omega, \gamma}(n)\right), \quad n \rightarrow \infty
\end{aligned}
$$

Putting (4.20)-(4.23) together, we get (4.18).

\section{Simple EXAMPLES}

We analyze perturbations of several simple operators. First in Section 5.1, following the constructions in [2, Sec.6.3] and [3, Sec.8.1], we provide examples of self-adjoint operators $A$ and perturbations $B$ showing that the condition $2 \alpha+\gamma>1$ cannot be weakened to $2 \alpha+\gamma=1$. Next, we consider perturbations of finite band infinite matrices and $-\mathrm{d}^{2} / \mathrm{d} x^{2}$ on a finite interval with Neumann boundary conditions, see Sections 5.2, 5.3, respectively. In both subsections, we give conditions on the self-adjoint operator and the perturbations guaranteeing that the assumptions (1.9) and (1.10) hold and thus the results of Section 3 are applicable. In particular, the eigensystem of the perturbed operator $T$ contains a Riesz basis of the underlying Hilbert space.

Some of the conclusions in Sections 5.2, 5.3 are certainly not new, see e.g. [1] for eigenvalue analysis of tridiagonal matrices, [12, Chap.XIX.3] or [25] for Riesz basis property of perturbations of $-\mathrm{d}^{2} / \mathrm{d} x^{2}$ in boundary conditions or $[8,9,10]$ for perturbations by singular potentials; nevertheless, the goal of these sections is to demonstrate the flexibility of our approach. For instance, $-\mathrm{d}^{2} / \mathrm{d} x^{2}$ with an infinite number of complex $\delta$-interactions can be treated with the same amount of effort as the perturbation by a bounded potential.

5.1. Optimality of condition (1.10). Consider $\mathcal{H}=\ell^{2}(\mathbb{N})$, its standard basis $\left\{e_{k}\right\}$ and define $A e_{k}:=\mu_{k} e_{k}, k \in \mathbb{N}$, where $\mu_{k}=k^{\gamma}, \gamma>0$. We denote by

$$
d_{k}:=\frac{1}{2}\left(\mu_{2 k}-\mu_{2 k-1}\right)=2^{\gamma-2} k^{\gamma-1}\left(\gamma+\mathcal{O}\left(k^{-1}\right)\right), \quad k \rightarrow \infty
$$

and define the perturbation

$$
B e_{2 k-1}:=-d_{k} t_{k} e_{2 k}, \quad B e_{2 k}:=d_{k} t_{k} e_{2 k-1}, \quad k \in \mathbb{N},
$$

where $\left\{t_{k}\right\} \subset(0,1)$ and $t_{k} \rightarrow 1$ as $k \rightarrow \infty$. 
While condition (1.9) is clearly satisfied, regarding (1.10) we get

$$
\left|\left\langle B e_{m}, e_{n}\right\rangle\right| \leq \min \left\{\left\|B e_{m}\right\|,\left\|B e_{n}\right\|\right\} \leq\left\|B e_{m}\right\|^{\frac{1}{2}}\left\|B e_{n}\right\|^{\frac{1}{2}} \leq C(m n)^{\frac{\gamma-1}{2}},
$$

so $\alpha=(1-\gamma) / 2$; hence $2 \alpha+\gamma=1$. We show below by elementary explicit calculations that the eigensystem of $T:=A+B$ does not contain even a basis.

Since the perturbation $B$ is block-diagonal, it suffices to analyze the 2-dimensional blocks corresponding to $\operatorname{span}\left\{e_{2 k-1}, e_{2 k}\right\}$,

$$
T_{k}:=A_{k}+B_{k}=\left(\begin{array}{cc}
\mu_{2 k-1}+d_{k} & 0 \\
0 & \mu_{2 k-1}+d_{k}
\end{array}\right)+d_{k}\left(\begin{array}{cc}
-1 & t_{k} \\
-t_{k} & 1
\end{array}\right) .
$$

Eigenvalues of $T_{k}$ can be of course calculated explicitly, see [2, Sec.6.3] and [3, Sec.8.1] for details, namely

$$
\begin{aligned}
T_{k} g_{k}^{ \pm} & =\left(\mu_{2 k-1}+d_{k} \pm d_{k} \tau_{k}\right) g_{k}^{ \pm}, \\
\tau_{k} & =\sqrt{1-t_{k}^{2}}, \quad g_{k}^{ \pm}=\left(\begin{array}{c}
1 \\
G_{k}^{ \pm}
\end{array}\right), \quad G_{k}=\left(\frac{1+\tau_{k}}{1-\tau_{k}}\right)^{\frac{1}{2}} .
\end{aligned}
$$

The norms of spectral projections $P_{k}^{ \pm}$of $T$ related to eigenvalues $\mu_{2 k-1}+d_{k} \pm d_{k} \tau_{k}$ are explicit as well. We denote by $\left\{\left(g_{k}^{ \pm}\right)^{*}\right\}$ the biorthonormal vectors to $\left\{g_{k}^{ \pm}\right\}$, i.e. $\left\langle g_{k}^{\nu},\left(g_{k}^{\mu}\right)^{*}\right\rangle=\delta_{\nu, \mu}$, then

$$
\left\|P_{k}^{ \pm}\right\|=\left\|\left\langle\cdot,\left(g_{k}^{ \pm}\right)^{*}\right\rangle g_{k}^{ \pm}\right\|=\left\|\left(g_{k}^{ \pm}\right)^{*}\right\|\left\|g_{k}^{ \pm}\right\|=\frac{1}{\tau_{k}^{2}}=\frac{1}{1-t_{k}^{2}} .
$$

Hence for $\left\{t_{k}\right\}, t_{k} \rightarrow 1$, the eigensystem of $T$ does not contain a basis of $\ell^{2}(\mathbb{N})$.

5.2. Finite band infinite matrices. Let $\mathcal{H}=\ell^{2}(\mathbb{N}), \gamma>0$ and

$$
A=\left(\begin{array}{ccccc}
a_{1} & 0 & 0 & 0 & . \\
0 & a_{2} & 0 & 0 & . \\
0 & 0 & a_{3} & 0 & . \\
0 & 0 & 0 & a_{4} & . \\
. & . & . & . & .
\end{array}\right), \quad B=\left(\begin{array}{ccccc}
b_{1}^{(0)} & b_{1}^{(1)} & 0 & 0 & . \\
b_{1}^{(-1)} & b_{2}^{(0)} & b_{2}^{(1)} & 0 & . \\
0 & b_{2}^{(-1)} & b_{3}^{(0)} & b_{3}^{(1)} & . \\
0 & 0 & b_{3}^{(-1)} & b_{4}^{(0)} & . \\
. & . & . & . & .
\end{array}\right),
$$

where, with some $M>0$,

$$
a_{k}=k^{\gamma}, \quad\left|b_{k}^{(j)}\right| \leq M k^{\omega}, \quad j \in\{-1,0,1\}, k \in \mathbb{N} .
$$

Clearly, $A$ considered with the maximal domain satisfies assumption (1.9) with $\psi_{k}=e_{k}, k \in \mathbb{N}$, where $\left\{e_{k}\right\}$ is the standard basis of $\ell^{2}(\mathbb{N})$, and $\mu_{k}=k^{\gamma}, k \in \mathbb{N}$. The form $b=\langle B \cdot, \cdot\rangle$, generated by $B$, satisfies

$$
\left|b\left(\psi_{m}, \psi_{n}\right)\right| \leq C \min \left\{m^{\omega}, n^{\omega}\right\} \leq C m^{\frac{\omega}{2}} n^{\frac{\omega}{2}},
$$

where $C>0$ is independent of $m, n$. Thus, condition (1.10) is satisfied if

$$
\omega<\gamma-1
$$

The tri-diagonal perturbation $B$ can be replaced by a finite band matrix with offdiagonal sequences $\left\{b_{k}^{(j)}\right\}_{k=1}^{\infty}, j \in\left\{-j_{0}, \ldots, j_{0}\right\}$, satisfying

$$
\left|b_{k}^{(j)}\right| \leq M k^{\omega}, \quad j \in\left\{-j_{0}, \ldots, j_{0}\right\}, k \in \mathbb{N}
$$

and it is easy to see that condition (1.10) is satisfied if (5.10) holds. 
5.3. Perturbations of $-\mathrm{d}^{2} / \mathrm{d} x^{2}$ on a finite interval with Neumann boundary conditions. Let $l \in(0, \infty)$ and consider the self-adjoint operator $A$ and the associated form $a$

$$
\begin{aligned}
A & =-\frac{\mathrm{d}^{2}}{\mathrm{~d} x^{2}}, \quad \operatorname{Dom}(A)=\left\{\psi \in W^{2,2}(-l, l): \psi^{\prime}( \pm l)=0\right\}, \\
a[\psi] & =\left\|\psi^{\prime}\right\|^{2}, \quad \operatorname{Dom}(a)=\left\{\psi \in W^{1,2}(-l, l)\right\} .
\end{aligned}
$$

Eigenvalues of $A$ and related orthonormal eigenfunctions read

$$
\mu_{k}=\left(\frac{k \pi}{2 l}\right)^{2}, \quad k \in \mathbb{N}_{0}, \quad \psi_{k}(x)= \begin{cases}\frac{1}{\sqrt{2 l}}, & k=0, \\ \frac{1}{\sqrt{l}} \cos \left(\sqrt{\mu_{k}}(x+l)\right), & k \in \mathbb{N},\end{cases}
$$

thus condition (1.9) is satisfied with $\gamma=2$. We analyze several perturbations of this $A$, in boundary conditions, by $\delta$-interactions, by $L^{1}$ and singular potentials. Results and proofs for perturbations of $-\mathrm{d}^{2} / \mathrm{d} x^{2}$ with Dirichlet boundary are completely analogous.

5.3.1. Robin boundary conditions. Consider the form

$$
b_{\mathrm{R}}[\psi]:=\nu_{+}|\psi(l)|^{2}-\nu_{-}|\psi(-l)|^{2}, \quad \nu_{ \pm} \in \mathbb{C}, \quad \psi \in \operatorname{Dom}(a) .
$$

The m-sectorial operator $T_{\mathrm{R}}$ associated with the form $t:=a+b_{\mathrm{R}}$ is actually $-\mathrm{d}^{2} / \mathrm{d} x^{2}$ with Robin boundary conditions

$$
T_{\mathrm{R}}=-\frac{\mathrm{d}^{2}}{\mathrm{~d} x^{2}}, \quad \operatorname{Dom}\left(T_{\mathrm{R}}\right)=\left\{\psi \in W^{2,2}(-l, l): \psi^{\prime}( \pm l)+\nu_{ \pm} \psi( \pm l)=0,\right\}
$$

see e.g. [21, Ex.VI.2.16]. Since $\left\{\psi_{m}\right\}$ from (5.13) are uniformly bounded, we have $\sup _{m, n \in \mathbb{N}_{0}}\left|b_{\mathrm{R}}\left(\psi_{m}, \psi_{n}\right)\right|<\infty$, thus $b_{\mathrm{R}}$ satisfies condition (1.10) with $\alpha=0$.

5.3.2. $\delta$-interactions. The $\delta$-potential placed at $x_{0}$ with a complex coupling $\nu$ generates the form

$$
b_{\delta}[\psi]:=\nu\left|\psi\left(x_{0}\right)\right|^{2}, \quad \nu \in \mathbb{C}, \quad x_{0} \in(-l, l), \quad \psi \in \operatorname{Dom}(a) .
$$

It satisfies condition (1.10) with $\alpha=0$ since $\left|b_{\delta}\left(\psi_{m}, \psi_{n}\right)\right| \leq|\nu| / l$, see (5.13). In fact, condition (1.10) is satisfied with $\alpha=0$ also for an infinite number of $\delta$ 's

$$
\sum_{k=1}^{\infty} \nu_{k} \delta\left(x-x_{k}\right), \quad\left\{\nu_{k}\right\} \in \ell^{1}(\mathbb{N}), \quad\left\{x_{k}\right\} \subset(-l, l) .
$$

Indeed, the corresponding form reads

$$
b_{\delta}^{\infty}[\psi]:=\sum_{k=1}^{\infty} \nu_{k}\left|\psi\left(x_{k}\right)\right|^{2}, \quad\left\{\nu_{k}\right\} \in \ell^{1}(\mathbb{N}), \quad\left\{x_{k}\right\} \subset(-l, l), \quad \psi \in \operatorname{Dom}(a)
$$

and we have $\left|b_{\delta}^{\infty}\left(\psi_{m}, \psi_{n}\right)\right| \leq\|\nu\|_{\ell^{1}(\mathbb{N})} / l$, see $(5.13)$.

5.3.3. $L^{1}$-potential. A function $V \in L^{1}(-l, l)$ generates the form

$$
b_{V}[\psi]:=\int_{-l}^{l} V(x)|\psi(x)|^{2} \mathrm{~d} x, \quad \psi \in \operatorname{Dom}(a) .
$$

Since $\left|b_{V}\left(\psi_{m}, \psi_{n}\right)\right| \leq\|V\|_{L^{1}(-l, l)} / l$, see (5.13), condition (1.10) is satisfied with $\alpha=0$. Notice that the classical formula is recovered by using Riemann-Lebesgue 
lemma, namely, the first correction $\lambda_{n}^{(1)}$ to eigenvalues of $A$, see Theorem 3.2, reads

$$
\begin{aligned}
\lambda_{n}^{(1)} & =\frac{1}{l} \int_{-l}^{l} V(x) \cos ^{2}\left(\sqrt{\mu_{n}}(x+l)\right) \mathrm{d} x \\
& =\frac{1}{2 l} \int_{-l}^{l} V(x) \mathrm{d} x+\frac{1}{2 l} \int_{-l}^{l} V(x) \cos \left(2 \sqrt{\mu_{n}}(x+l)\right) \mathrm{d} x \\
& =\frac{1}{2 l} \int_{-l}^{l} V(x) \mathrm{d} x+o(1), \quad n \rightarrow \infty .
\end{aligned}
$$

5.3.4. Singular potentials. Consider $V \in W^{-s, 2}(-l, l)$ with some $s \geq 0$, so

$$
\exists C>0, \exists s \geq 0, \forall \phi \in W^{1,2}(-l, l),|(V, \phi)| \leq C\|\phi\|_{W^{1,2}(-l, l)}^{s}\|\phi\|^{1-s} .
$$

The associated form, a generalization of (5.19), reads

$$
b_{V}(\phi, \psi):=(V, \phi \bar{\psi}), \quad \phi, \psi \in \operatorname{Dom}(a) .
$$

For $\left\{\psi_{m}\right\}$ from (5.13), we have $\left\|\psi_{m}\right\|=\mathcal{O}(1)$ and $\left\|\psi_{m}^{\prime}\right\|=\mathcal{O}(m)$ as $m \rightarrow \infty$, thus we get from (5.21) that

$$
\begin{aligned}
\left|b_{V}\left(\psi_{m}, \psi_{n}\right)\right| & =\left|\left(V, \psi_{m} \psi_{n}\right)\right| \leq C\left(\left\|\left(\psi_{m} \psi_{n}\right)^{\prime}\right\|^{2}+\left\|\psi_{m} \psi_{n}\right\|^{2}\right)^{\frac{s}{2}}\left\|\psi_{m} \psi_{n}\right\|^{1-s} \\
& \leq C_{1}(m+n)^{s} \leq C_{2}(m n)^{s} .
\end{aligned}
$$

Hence $b_{V}$ satisfies condition (1.10) if $s<1 / 2$.

\section{Perturbations of Single-well Schrödinger operators}

Our main examples are perturbations of self-adjoint Schrödinger operators $A$ in $L^{2}(\mathbb{R})$ with the associated quadratic forms $a$

$$
\begin{gathered}
A=-\frac{\mathrm{d}^{2}}{\mathrm{~d} x^{2}}+Q(x), \quad \operatorname{Dom}(A)=\left\{\psi \in W^{2,2}(\mathbb{R}): Q \psi \in L^{2}(\mathbb{R})\right\}, \\
a[\psi]=\left\|\psi^{\prime}\right\|^{2}+\left\|Q^{\frac{1}{2}} \psi\right\|^{2}, \quad \operatorname{Dom}(a)=\left\{\psi \in W^{1,2}(\mathbb{R}): Q^{\frac{1}{2}} \psi \in L^{2}(\mathbb{R})\right\} .
\end{gathered}
$$

The real potential $Q$ is assumed to satisfy the following.

Assumption I. Suppose that $Q \in C^{2}(\mathbb{R}) \cap C^{3}(\mathbb{R} \backslash[-l, l])$ with some $l \geq 0, Q \geq 0$, $Q(0)=0, Q$ is even, increasing on $(0, \infty), Q^{\prime}$ is non-decreasing on $(0, \infty)$,

$$
\frac{Q^{(k+1)}(x)}{Q^{(k)}(x)}=\mathcal{O}\left(\frac{1}{x}\right), \quad x \rightarrow+\infty, \quad k=0,1,2,
$$

and

$$
\exists \beta>1, \quad \lim _{x \rightarrow \infty} \frac{Q(x)}{|x|^{\beta}}=1 .
$$

Under Assumption I, Schrödinger operator $A$ in (6.1) is self-adjoint, bounded from below and has a compact resolvent; see e.g. [32, Thm.XII.67].

Recall that (6.2) with $k=0$ and Gronwall's inequality imply that $Q$ cannot grow faster than a polynomial, nonetheless, we assume the precise behavior at infinity in (6.3). Moreover, (6.2) implies further that, for every $\sigma \in(0, \infty)$ and $k \in\{0,1,2\}$

$$
\frac{Q^{(k)}(\sigma x)}{Q^{(k)}(x)}=\mathcal{O}(1), \quad x \rightarrow \infty,
$$

see $[38, \S 22.27]$. For $k=0,(6.4)$ follows from (in the non-obvious case $\sigma>1$ )

$$
\log \frac{Q(\sigma x)}{Q(x)}=\int_{x}^{\sigma x} \frac{Q^{\prime}(t)}{Q(t)} \mathrm{d} t=\mathcal{O}\left(\int_{x}^{\sigma x} \frac{\mathrm{d} s}{s}\right)=\mathcal{O}(1), \quad x \rightarrow \infty
$$


the other cases are similar. The additional condition (6.3) implies more; namely, for every $\sigma \in(0, \infty)$,

$$
\lim _{x \rightarrow+\infty} \frac{Q(\sigma x)}{Q(x)}=\sigma^{\beta} \lim _{x \rightarrow+\infty} \frac{Q(\sigma x)}{(\sigma x)^{\beta}} \frac{x^{\beta}}{Q(x)}=\sigma^{\beta} .
$$

Recall also that since $Q^{\prime}$ is non-decreasing on $(0, \infty)$, we have

$$
Q(y)-Q(x) \leq Q^{\prime}(y)(y-x), \quad x, y>0 .
$$

We define the (positive) turning points $x_{\mu}$ and the associated quantity $a_{\mu}$ for $Q^{\prime}$ by relations

$$
Q\left(x_{\mu}\right)=\mu, \quad x_{\mu}>0, \quad a_{\mu}:=Q^{\prime}\left(x_{\mu}\right) .
$$

Notice that the assumption (6.3) implies

$$
\lim _{\mu \rightarrow+\infty} \frac{\mu^{\frac{1}{\beta}}}{x_{\mu}}=\lim _{\mu \rightarrow+\infty}\left(\frac{Q\left(x_{\mu}\right)}{x_{\mu}^{\beta}}\right)^{\frac{1}{\beta}}=1 .
$$

6.1. Eigenvalues of $A$. The spectrum of $A$ contains only simple discrete eigenvalues $\left\{\mu_{k}\right\}_{k \in \mathbb{N}} \subset \mathbb{R}_{+}$which are known to obey, see [39, Sec.7.7],

$$
\int_{-x_{\mu_{k}}}^{x_{\mu_{k}}}\left(\mu_{k}-Q(x)\right)^{\frac{1}{2}} \mathrm{~d} x=\left(k+\frac{1}{2}\right) \pi+\mathcal{O}\left(k^{-1}\right), \quad k \rightarrow \infty .
$$

The result (6.10) holds also if (6.3) is replaced by

$$
\lim _{x \rightarrow \infty} Q(x)=+\infty \text {. }
$$

More can be said under the additional condition (6.3).

Proposition 6.1. Let $Q$ satisfy Assumption I. Then the eigenvalues $\left\{\mu_{k}\right\}$ of the operator $A$ in (6.1) satisfy

$$
\begin{array}{rlrl}
\mu_{k} & =\left(\frac{\pi}{\Omega_{\beta}} k\right)^{\gamma}(1+o(1)), & k \rightarrow \infty, \\
\mu_{k+1}-\mu_{k}=\frac{2 \pi}{\Omega_{\beta}^{\prime}}\left(\frac{\pi}{\Omega_{\beta}} k\right)^{\gamma-1}(1+o(1)), & k \rightarrow \infty,
\end{array}
$$

where

$$
\gamma=\frac{2 \beta}{\beta+2}, \quad \Omega_{\beta}=2 \int_{0}^{1}\left(1-t^{\beta}\right)^{\frac{1}{2}} \mathrm{~d} t, \quad \Omega_{\beta}^{\prime}=2 \int_{0}^{1} \frac{\mathrm{d} t}{\left(1-t^{\beta}\right)^{\frac{1}{2}}} .
$$

Before we give the proof of Proposition 6.1 we show two following.

Lemma 6.2. Let $Q$ satisfy Assumption I. Then

$$
\begin{aligned}
& \lim _{x \rightarrow+\infty} 2 \int_{0}^{1}\left(1-\frac{Q(x t)}{Q(x)}\right)^{\frac{1}{2}} \mathrm{~d} t=\Omega_{\beta}, \\
& \lim _{x \rightarrow+\infty} 2 \int_{0}^{1}\left(1-\frac{Q(x t)}{Q(x)}\right)^{-\frac{1}{2}} \mathrm{~d} t=\Omega_{\beta}^{\prime} .
\end{aligned}
$$

Proof. Since $Q$ is increasing, we have $Q(x t) / Q(x) \leq 1$ for all $t \in[0,1]$, thus the dominated convergence theorem and (6.6) justifies the first limit in (6.15).

To show the second limit, we analyze separately the cases $0 \leq t \leq 1 / 2$ and $1 / 2 \leq t \leq 1$. For the former, we have from (6.6) that

$$
\left(1-\frac{Q(x t)}{Q(x)}\right)^{-\frac{1}{2}} \leq\left(1-\frac{Q\left(\frac{x}{2}\right)}{Q(x)}\right)^{-\frac{1}{2}} \rightarrow\left(1-2^{-\beta}\right)^{-\frac{1}{2}}
$$


for the latter, we get from the mean value theorem, (6.7) with $Q(0)=0$ and (6.6) that

$$
\begin{aligned}
\left(1-\frac{Q(x t)}{Q(x)}\right)^{-\frac{1}{2}} & \leq\left(\frac{Q(x)}{Q^{\prime}(x t) x(1-t)}\right)^{\frac{1}{2}} \leq\left(\frac{Q(x)}{Q^{\prime}\left(\frac{x}{2}\right) x(1-t)}\right)^{\frac{1}{2}} \\
& \leq\left(\frac{Q(x)}{2 Q\left(\frac{x}{2}\right)(1-t)}\right)^{\frac{1}{2}} \rightarrow \frac{2^{\frac{\beta-1}{2}}}{(1-t)^{\frac{1}{2}}} .
\end{aligned}
$$

Hence there is a constant $C>0$ such that for all sufficiently large $x$ and all $0<t<1$

$$
\left(1-\frac{Q(x t)}{Q(x)}\right)^{-\frac{1}{2}} \leq \frac{C}{(1-t)^{\frac{1}{2}}},
$$

which is integrable on $(0,1)$ and the second limit in $(6.15)$ is justified by the dominated convergence theorem and (6.6).

Proof of Proposition 6.1. Simple manipulations with (6.10) leads to

$$
2 \mu_{k}^{\frac{1}{2}} x_{\mu_{k}} \int_{0}^{1}\left(1-\frac{Q\left(x_{\mu_{k}} t\right)}{Q\left(x_{\mu_{k}}\right)}\right)^{\frac{1}{2}} \mathrm{~d} t=\pi k(1+o(1)), \quad k \rightarrow \infty .
$$

Hence (6.12) follows from (6.19), (6.9) and (6.15).

To show (6.13), we define the function

$$
g(\mu):=\int_{0}^{x_{\mu}}(\mu-Q(x))^{\frac{1}{2}} \mathrm{~d} x
$$

and start with the identity obtained from (6.10)

$$
g\left(\mu_{k+1}\right)-g\left(\mu_{k}\right)=\frac{\pi}{2}(1+o(1)), \quad k \rightarrow \infty .
$$

Observing that

$$
\lim _{x \rightarrow x_{\mu}} \frac{x_{\mu}-x}{\mu-Q(x)}=\frac{1}{a_{\mu}},
$$

we can check that $g$ is differentiable and, after a change of variables,

$$
g^{\prime}(\mu)=\frac{x_{\mu}}{2 \mu^{\frac{1}{2}}} \int_{0}^{1}\left(1-\frac{Q(x t)}{Q(x)}\right)^{-\frac{1}{2}} \mathrm{~d} t .
$$

The mean value theorem yields (with $\eta_{k} \in\left(\mu_{k}, \mu_{k+1}\right)$ )

$$
g\left(\mu_{k+1}\right)-g\left(\mu_{k}\right)=g^{\prime}\left(\eta_{k}\right)\left(\mu_{k+1}-\mu_{k}\right),
$$

and therefore, using (6.21), (6.23), (6.15), (6.6) and (6.9), we obtain

$$
\mu_{k+1}-\mu_{k}=\frac{\pi}{2 g^{\prime}\left(\eta_{k}\right)}(1+o(1))=\frac{2 \pi}{\Omega_{\beta}^{\prime}} \mu_{k}^{\frac{\beta-2}{2 \beta}}(1+o(1)) .
$$

Then (6.13) follows by employing (6.12).

6.2. Eigenfunctions of $A$ and their weighted $L^{q}$-norms. Since $Q$ is even, orthonormal eigenfunctions $\left\{\psi_{k}\right\}$, related to eigenvalues $\left\{\mu_{k}\right\}$, are even or odd functions. Moreover, even with (6.3) replaced by (6.11), $\left\{\psi_{k}\right\}$ can be chosen such that they satisfy (see e.g. $[38, \S 22.27]$ and [15])

$$
\psi_{k}(x)=\frac{1}{\left\|u_{k}\right\|} u_{k}(x)\left(1+\mathcal{O}\left(x_{\mu_{k}}^{-1} \mu_{k}^{-\frac{1}{2}}\right)\right), \quad x>0,
$$


where $u_{k}=u\left(x, \mu_{k}\right)$ with

$$
\begin{aligned}
& u=u(x, \mu)=\left(\frac{\zeta}{\zeta^{\prime}}\right)^{\frac{1}{2}} K_{\frac{1}{3}}(-i \zeta), \\
& \zeta=\zeta(x, \mu)= \begin{cases}\int_{x}^{x_{\mu}}(\mu-Q(s))^{\frac{1}{2}} \mathrm{~d} s, & 0<x<x_{\mu}, \\
i \int_{x_{\mu}}^{x}(Q(s)-\mu)^{\frac{1}{2}} \mathrm{~d} s, & x>x_{\mu} ;\end{cases}
\end{aligned}
$$

$K_{\frac{1}{3}}$ is the modified Bessel function of order $1 / 3$. Using the asymptotic formulas for Bessel functions, we get further that, see e.g. [15],

$$
u^{2}(x)=\frac{\pi}{(\mu-Q(x))^{\frac{1}{2}}}\left(1+\sin 2 \zeta+R_{1}(\zeta)\right), \quad \zeta>1,
$$

where $\left|R_{1}(\zeta)\right|<1 /(2 \zeta)$, and

$$
|u(x)| \leq \begin{cases}\frac{A_{1}}{(\mu-Q(x))^{\frac{1}{4}}}, & 0 \leq x<x_{\mu}-\delta, \\ \frac{A_{1}}{\left(\mu-Q\left(x_{\mu}-\delta\right)\right)^{\frac{1}{4}}}, & x_{\mu}-\delta \leq x \leq x_{\mu}+\delta_{1}, \\ \frac{A_{1}}{2(Q(x)-\mu)^{\frac{1}{4}}} e^{-\int_{x_{\mu}}^{x}(Q(s)-\mu)^{\frac{1}{2}} \mathrm{~d} s}, & x>x_{\mu}+\delta_{1},\end{cases}
$$

where $A_{1}=2.7$ and numbers $\delta, \delta_{1}$ are defined by equations

$$
\zeta\left(x_{\mu}-\delta\right)=-i \zeta\left(x_{\mu}+\delta_{1}\right)=1 .
$$

It can be shown, see e.g. the appendix of [30], that

$$
\delta, \delta_{1}=\mathcal{O}\left(a_{\mu}^{-\frac{1}{3}}\right), \quad \delta^{-1}, \delta_{1}^{-1}=\mathcal{O}\left(a_{\mu}^{\frac{1}{3}}\right) .
$$

Further, it is known, see [15, Lemma 5], that

$$
\int_{0}^{\infty} u^{2}(x) \mathrm{d} x=\int_{0}^{x_{\mu}} \frac{\pi \mathrm{d} x}{(\mu-Q(x))^{\frac{1}{2}}}\left(1+\mathcal{O}\left(x_{\mu}^{-\frac{1}{3}} \mu^{-\frac{1}{6}}\right)\right) .
$$

Under the assumption (6.3) we therefore obtain from (6.15) and (6.9) that

$$
\left\|u_{k}\right\|^{2}=\pi \Omega_{\beta}^{\prime} \mu_{k}^{\frac{2-\beta}{2 \beta}}(1+o(1))=\pi \Omega_{\beta}^{\prime}\left(\frac{\pi}{\Omega_{\beta}} k\right)^{\frac{2-\beta}{2+\beta}}(1+o(1)), \quad k \rightarrow \infty,
$$

where $\Omega_{\beta}, \Omega_{\beta}^{\prime}$ are as in (6.14).

Finally, we recall the pointwise estimates for $\left\{\psi_{k}\right\}$, see [39, Sec.8],

$$
\begin{aligned}
\left|\psi_{k}(x)\right| & =\mathcal{O}\left(x_{\mu_{k}^{-\frac{1}{2}}}\right)=\mathcal{O}\left(k^{-\frac{1}{\beta+2}}\right), \\
\left|\psi_{k}^{\prime}(x)\right| & =\mathcal{O}\left(\mu_{k}^{\frac{1}{2}} x_{\mu_{k}}^{-\frac{1}{2}}\right)=\mathcal{O}\left(k^{\frac{\beta-1}{\beta+2}}\right), \\
\left\|\psi_{k}\right\|_{L^{\infty}(\mathbb{R})} & =\mathcal{O}\left(\mu_{k}^{\frac{1}{4}} x_{\mu_{k}^{-\frac{1}{2}}}^{-\frac{1}{6}} a_{\mu_{k}^{6}}\right)=\mathcal{O}\left(k^{\frac{\beta-4}{6(\beta+2)}}\right), \quad k \rightarrow \infty ;
\end{aligned}
$$

the first equalities hold also if (6.3) is replaced by (6.11); notice that the point $x \in \mathbb{R}$ is arbitrary but fixed in (6.35) and (6.36).

Next, we estimate the weighted $L^{q}$-norms of $\left\{\psi_{k}\right\}$. For $\beta=2$ and without the weight, we recover the known results for Hermite functions, see [37, Lemma 1.5.2], where in fact both-sided estimates are given. For $q=2$, and $Q, w$ satisfying similar conditions like here, both-sided estimates (in fact limits) are established in [30]. 
Proposition 6.3. Let $Q$ satisfy Assumption I with (6.3) replaced by (6.11), let $x_{\mu}$, $a_{\mu}$ be as in (6.8) and let $\left\{\psi_{k}\right\}$ be as in (6.26). Suppose that the weight $w \in C^{1}(\mathbb{R})$ is positive, even, increasing on $(0, \infty)$ and satisfy

$$
\frac{w^{\prime}(x)}{w(x)}=\mathcal{O}\left(\frac{1}{x}\right), \quad x \rightarrow \infty
$$

Then

$$
\left\|w \psi_{k}\right\|_{L^{q}(\mathbb{R})}=\mathcal{O}\left(w\left(x_{\mu_{k}}\right)\left\|\psi_{k}\right\|_{L^{q}(\mathbb{R})}\right), \quad k \rightarrow \infty .
$$

Furthermore, as $k \rightarrow \infty$,

$$
\left\|\psi_{k}\right\|_{L^{q}(\mathbb{R})}= \begin{cases}\mathcal{O}\left(\left(a_{\mu_{k}} \mu_{k}^{-1}\right)^{\frac{q-2}{2 q}}\right), & 1 \leq q<4, \\ \mathcal{O}\left(\left(a_{\mu_{k}} \mu_{k}^{-1}\right)^{-\frac{1}{4}}\left(\log \left(\mu_{k} a_{\mu_{k}}^{-\frac{2}{3}}\right)\right)^{\frac{1}{4}}\right), & q=4, \\ \mathcal{O}\left(a_{\mu_{k}}^{\frac{q-1}{3 q}} \mu_{k}^{-\frac{1}{4}}\right), & q>4 .\end{cases}
$$

If (6.3) is satisfied in addition, then, as $k \rightarrow \infty$,

$$
\left\|\psi_{k}\right\|_{L^{q}(\mathbb{R})}= \begin{cases}\mathcal{O}\left(k^{\frac{2-q}{q(\beta+2)}}\right), & 1 \leq q<4 \\ \mathcal{O}\left(k^{-\frac{1}{2(\beta+2)}}(\log k)^{\frac{1}{4}}\right), & q=4 \\ \mathcal{O}\left(k^{\frac{4-4 \beta-4 q+q \beta}{6 q(\beta+2)}}\right), & q>4 .\end{cases}
$$

Proof. We suppress the subscript $k$ in the sequel and keep $\mu$ only. The letter $C$ denotes a constant, which can vary in every step, however, it is independent of $\mu$. The case $q=\infty$ is reduces to (6.37), so we analyze $1 \leq q<\infty$ only.

Since $Q^{\prime}$ is non-decreasing on $(0, \infty)$, we get from $(6.33)$ that

$$
\int_{0}^{\infty} u^{2} \mathrm{~d} x \geq C \int_{0}^{x_{\mu}} \frac{\mathrm{d} x}{(\mu-Q)^{\frac{1}{2}}} \geq \frac{1}{a_{\mu}} \int_{0}^{x_{\mu}} \frac{Q^{\prime} \mathrm{d} x}{(\mu-Q)^{\frac{1}{2}}}=\frac{\mu^{\frac{1}{2}}}{a_{\mu}} .
$$

Thus, we have from (6.26) and (6.42) that

$$
\|w \psi\|_{L^{q}(\mathbb{R})} \leq C\|u\|^{-1}\|w u\|_{L^{q}(\mathbb{R})} \leq C a_{\mu}^{\frac{1}{2}} \mu^{-\frac{1}{4}}\|w u\|_{L^{q}(\mathbb{R})} .
$$

In the following, we split the integral and employ (6.30) in estimates,

$$
\int_{0}^{\infty}|w u|^{q} \mathrm{~d} x=\left(\int_{0}^{x_{\frac{\mu}{2}}}+\int_{x_{\frac{\mu}{2}}}^{x_{\mu}-\delta}+\int_{x_{\mu}-\delta}^{x_{\mu}+\delta_{1}}+\int_{x_{\mu}+\delta_{1}}^{x_{\frac{3}{2} \mu}}+\int_{x_{\frac{3}{2} \mu}}^{\infty}\right)|w u|^{q} \mathrm{~d} x .
$$

- $x<x_{\frac{\mu}{2}}$ : For all sufficiently large $\mu$, we have $x_{\frac{\mu}{2}} \leq x_{\mu}-\delta$. To see this, we use that $Q$ is increasing, $Q\left(x_{\mu / 2}\right)=\mu / 2$ and by the mean value theorem and $Q^{\prime}(x) / Q(x)=\mathcal{O}(1 / x)$ we get

$$
\frac{Q\left(x_{\mu}-\delta\right)}{Q\left(x_{\mu}\right)}=1-\frac{Q\left(x_{\mu}\right)-Q\left(x_{\mu}-\delta\right)}{Q\left(x_{\mu}\right)}=1+\mathcal{O}\left(\frac{\delta}{x_{\mu}}\right), \quad \mu \rightarrow \infty .
$$

Hence, using (6.30) and $Q^{\prime}(x) / Q(x)=\mathcal{O}(1 / x)$ in the last step, we obtain

$$
\int_{0}^{x \frac{\mu}{2}}|w u|^{q} \mathrm{~d} x \leq \frac{C w\left(x_{\mu}\right)^{q} x_{\mu}}{\left(\mu-Q\left(x_{\frac{\mu}{2}}\right)\right)^{\frac{q}{4}}} \leq C w\left(x_{\mu}\right)^{q} x_{\mu} \mu^{-\frac{q}{4}} \leq C w\left(x_{\mu}\right)^{q} a_{\mu}^{-1} \mu^{1-\frac{q}{4}} .
$$

- $x_{\frac{\mu}{2}}<x<x_{\mu}-\delta$ : since $Q^{\prime}$ is non-decreasing,

$$
\int_{x_{\frac{\mu}{2}}}^{x_{\mu}-\delta}|w u|^{q} \mathrm{~d} x \leq C w\left(x_{\mu}\right)^{q} a_{\frac{\mu}{2}}^{-1} \int_{x_{\frac{\mu}{2}}}^{x_{\mu}-\delta} \frac{Q^{\prime} \mathrm{d} x}{(\mu-Q)^{\frac{q}{4}}} .
$$


We can replace $a_{\frac{\mu}{2}}$ by $a_{\mu}$ since from (6.7) and $Q^{\prime}(x) / Q(x)=\mathcal{O}(1 / x)$, we get

$$
1 \geq \frac{a_{\frac{\mu}{2}}}{a_{\mu}} \geq \frac{Q\left(x_{\frac{\mu}{2}}\right)}{x_{\frac{\mu}{2}} Q^{\prime}\left(x_{\mu}\right)} \geq C \frac{Q\left(x_{\frac{\mu}{2}}\right) x_{\mu}}{x_{\frac{\mu}{2}} Q\left(x_{\mu}\right)} \geq \frac{C}{2} .
$$

Further,

$1 \leq q<4$ :

$$
\int_{x_{\frac{\mu}{2}}}^{x_{\mu}-\delta} \frac{Q^{\prime} \mathrm{d} x}{(\mu-Q)^{\frac{q}{4}}} \leq \frac{4-q}{4}\left(\mu-Q\left(x_{\frac{\mu}{2}}\right)\right)^{1-\frac{q}{4}} \leq C \mu^{1-\frac{q}{4}} .
$$

$q=4:$ by (6.4) and (6.32),

$$
\int_{x_{\frac{\mu}{2}}}^{x_{\mu}-\delta} \frac{Q^{\prime} \mathrm{d} x}{\mu-Q}=\log \frac{\mu}{2\left(Q\left(x_{\mu}\right)-Q\left(x_{\mu}-\delta\right)\right)} \leq C \log \frac{\mu}{a_{\mu} \delta} \leq C \log \left(\mu a_{\mu}^{-\frac{2}{3}}\right) .
$$

$q>4$ : again by $(6.4)$ and $(6.32)$,

$$
\int_{x_{\frac{\mu}{2}}}^{x_{\mu}-\delta} \frac{Q^{\prime} \mathrm{d} x}{(\mu-Q)^{\frac{q}{4}}} \leq \frac{q-4}{4\left(\mu-Q\left(x_{\mu}-\delta\right)\right)^{\frac{q}{4}-1}} \leq \frac{C}{\left(a_{\mu} \delta\right)^{\frac{q}{4}-1}} \leq C a_{\mu}^{\frac{2}{3}-\frac{q}{6}} .
$$

In summary,

$$
\int_{x_{\frac{\mu}{2}}}^{x_{\mu}-\delta}|w u|^{q} \mathrm{~d} x \leq C w\left(x_{\mu}\right)^{q} a_{\mu}^{-1} \begin{cases}\mu^{1-\frac{q}{4}}, & 1 \leq q<4, \\ \log \left(\mu a_{\mu}^{-\frac{2}{3}}\right), & q=4, \\ a_{\mu}^{\frac{2}{3}-\frac{q}{6}}, & q>4 .\end{cases}
$$

- $x_{\mu}-\delta<x<x_{\mu}+\delta_{1}$ : Notice that since $w$ satisfies (6.38), we have

$$
\forall \sigma \in(0, \infty), \quad w(\sigma x)=\mathcal{O}(w(x)), \quad x \rightarrow+\infty,
$$

see (6.4) and (6.5). Then by (6.30), (6.4) and (6.32)

$$
\int_{x_{\mu}-\delta}^{x_{\mu}+\delta_{1}}|w u|^{q} \mathrm{~d} x \leq C w\left(x_{\mu}\right)^{q} \frac{\delta+\delta_{1}}{Q^{\prime}\left(x_{\mu}-\delta\right)^{\frac{q}{4}} \delta^{\frac{q}{4}}} \leq C w\left(x_{\mu}\right)^{q} a_{\mu}^{-\frac{1}{3}-\frac{q}{6}} .
$$

- $x_{\mu}+\delta_{1}<x<x_{\frac{3}{2} \mu}$ : like for $x_{\frac{\mu}{2}}$, it can showed that $x_{\frac{3}{2} \mu} \geq x_{\mu}+\delta_{1}$ for all sufficiently large $\mu$. Then, using (6.30), we get

$$
\int_{x_{\mu}+\delta_{1}}^{x_{\frac{3}{2} \mu}}|w u|^{q} \mathrm{~d} x \leq C w\left(x_{\mu}\right)^{q} a_{\mu}^{-1} \int_{x_{\mu}+\delta_{1}}^{x_{\frac{3}{2} \mu}} \frac{Q^{\prime} \mathrm{d} x}{(Q-\mu)^{\frac{q}{4}}} ;
$$

here $w\left(x_{\frac{3}{2} \mu}\right)$ is replaced by $w\left(x_{\mu}\right)$ since we have $(6.53)$ and $x_{\frac{3}{2} \mu}=\mathcal{O}\left(x_{\mu}\right)$. To see the latter, we use that $Q^{\prime}$ is non-decreasing on $(0, \infty)$ and (6.7) with $Q(0)=0$,

$$
\frac{x_{\frac{3}{2} \mu}}{x_{\mu}}=\frac{x_{\mu}+Q^{-1}\left(\frac{3}{2} \mu\right)-Q^{-1}(\mu)}{x_{\mu}} \leq \frac{x_{\mu}+\frac{\mu}{2 a_{\mu}}}{x_{\mu}} \leq \frac{3}{2} .
$$

Further,

$1 \leq q<4$ :

$$
\int_{x_{\mu}+\delta_{1}}^{x_{\frac{3}{2} \mu}} \frac{Q^{\prime} \mathrm{d} x}{(Q-\mu)^{\frac{q}{4}}} \leq \frac{4-q}{4}\left(Q\left(x_{\frac{3}{2} \mu}\right)-\mu\right)^{1-\frac{q}{4}} \leq C \mu^{1-\frac{q}{4}} .
$$

$q=4:$ by $(6.32)$,

$$
\int_{x_{\mu}+\delta_{1}}^{x^{\frac{3}{2} \mu}} \frac{Q^{\prime} \mathrm{d} x}{Q-\mu}=\log \frac{\mu}{2\left(Q\left(x_{\mu}+\delta_{1}\right)-Q\left(x_{\mu}\right)\right)} \leq C \log \frac{\mu}{a_{\mu} \delta_{1}} \leq C \log \left(\mu a_{\mu}^{-\frac{2}{3}}\right) .
$$

$q>4$ : by $(6.4)$ and $(6.32)$,

$$
\int_{x_{\mu}+\delta_{1}}^{x_{\frac{3}{2} \mu}} \frac{Q^{\prime} \mathrm{d} x}{(Q-\mu)^{\frac{q}{4}}} \leq \frac{q-4}{4}\left(Q\left(x_{\mu}+\delta_{1}\right)-\mu\right)^{1-\frac{q}{4}} \leq C a_{\mu}^{\frac{2}{3}-\frac{q}{6}} .
$$


In summary,

$$
\int_{x_{\mu}+\delta_{1}}^{x_{\frac{3}{2} \mu}}|w u|^{q} \mathrm{~d} x \leq C w\left(x_{\mu}\right)^{q} a_{\mu}^{-1} \begin{cases}\mu^{1-\frac{q}{4}}, & 1 \leq q<4, \\ \log \left(\mu a_{\mu}^{-\frac{2}{3}}\right), & q=4, \\ a_{\mu}^{\frac{2}{3}-\frac{q}{6}}, & q>4 .\end{cases}
$$

- $x_{\frac{3}{2} \mu}<x$ : first, using $Q^{\prime}(x) / Q(x)=\mathcal{O}(1 / x)$, we get

$$
\int_{x_{\mu}}^{x}(Q-\mu)^{\frac{1}{2}} \mathrm{~d} s \geq \frac{2}{3} \frac{Q(x)^{\frac{3}{2}}}{Q^{\prime}(x)}\left(1-\frac{\mu}{Q(x)}\right)^{\frac{3}{2}} \geq C \mu^{\frac{1}{2}} x .
$$

Since $w$ does not grow faster than a polynomial, see (6.53) and Gronwall's inequality, we have from (6.30) that

$$
\int_{x_{\frac{3}{2} \mu}}^{\infty}|w(x) u(x)|^{q} \mathrm{~d} x \leq C \mu^{-\frac{q}{4}} \int_{x_{\frac{3}{2} \mu}}^{\infty} w(x) e^{-C \mu^{\frac{1}{2}} x} \mathrm{~d} x \leq e^{-C \mu^{\frac{1}{2}} x_{\mu}} .
$$

Putting all estimates from above together, we get

$$
\|w \psi\|_{L^{q}(\mathbb{R})} \leq C w\left(x_{\mu}\right)\left(a_{\mu}^{\frac{1}{3}-\frac{1}{3 q}} \mu^{-\frac{1}{4}}+a_{\mu}^{\frac{1}{2}-\frac{1}{q}} \mu^{-\frac{1}{2}+\frac{1}{q}} \iota_{q}(\mu)^{\frac{1}{q}}\right)
$$

where

$$
\iota_{q}(\mu):= \begin{cases}1, & q \neq 4 \\ \log \left(\mu a_{\mu}^{-\frac{2}{3}}\right), & q=4 .\end{cases}
$$

Finally, for $1 \leq q<4$,

$$
a_{\mu}^{\frac{1}{3}-\frac{1}{3 q}} \mu^{-\frac{1}{4}}+a_{\mu}^{\frac{1}{2}-\frac{1}{q}} \mu^{-\frac{1}{2}+\frac{1}{q}}=a_{\mu}^{\frac{1}{2}-\frac{1}{q}} \mu^{-\frac{1}{2}+\frac{1}{q}}\left(1+\left(a_{\mu}^{\frac{1}{6}} \mu^{-\frac{1}{4}}\right)^{\frac{4-q}{q}}\right)
$$

and, for $q>4$,

$$
a_{\mu}^{\frac{1}{3}-\frac{1}{3 q}} \mu^{-\frac{1}{4}}+a_{\mu}^{\frac{1}{2}-\frac{1}{q}} \mu^{-\frac{1}{2}+\frac{1}{q}}=a_{\mu}^{\frac{1}{3}-\frac{1}{3 q}} \mu^{-\frac{1}{4}}\left(1+\left(a_{\mu}^{\frac{1}{6}} \mu^{-\frac{1}{4}}\right)^{\frac{q-4}{q}}\right)
$$

thus (6.39) and (6.40) follow since $Q^{\prime}(x) / Q(x)=\mathcal{O}(1 / x)$ implies $a_{\mu} / \mu=\mathcal{O}\left(1 / x_{\mu}\right)$.

If (6.3) holds in addition, we obtain (6.41) from (6.40) by employing (6.9), (6.12), (6.7) and $Q^{\prime}(x) / Q(x)=\mathcal{O}(1 / x)$ as $x \rightarrow \infty$.

6.3. Perturbations by functional potentials $V$ in weighted $L^{p}$-spaces. We define the following spaces

$$
L(p, \tau):=\left\{v:\left(1+x^{2}\right)^{-\frac{\tau}{2}}|v(x)| \in L^{p}(\mathbb{R})\right\}, \quad 1 \leq p \leq \infty, \quad \tau \in \mathbb{R},
$$

as in $[2,29]$; notice that $L(p, 0)=L^{p}(\mathbb{R})$.

The form associated with the perturbation by a functional potential $V$ reads

$$
b_{V}[\psi]:=\int_{\mathbb{R}} V|\psi|^{2}, \quad \operatorname{Dom}\left(b_{V}\right):=\left\{\psi \in L^{2}(\mathbb{R}): V|\psi|^{2} \in L^{1}(\mathbb{R})\right\} .
$$

Theorem 6.4. Let $Q$ satisfy Assumption I and let $A$ be Schrödinger operator from (6.1). Suppose that $V \in L(p, \tau)$ with $1 \leq p \leq \infty, \tau \geq 0$, and, depending on $p$, one of the following conditions is satisfied

$$
\begin{array}{ll}
\tau<\frac{2}{3}(\beta-1)\left(1-\frac{1}{2 p}\right) & \text { if } 1 \leq p<2, \\
\tau<\frac{\beta-2}{2}+\frac{1}{p} & \text { if } \quad 2 \leq p \leq \infty .
\end{array}
$$


Then $A$ and the form $b_{V}$ from (6.68) satisfy conditions (1.9) and (1.10) with

$$
\gamma=\frac{2 \beta}{\beta+2}, \quad \alpha=\frac{1}{\beta+2} \begin{cases}\frac{\beta+2}{6}+\frac{1-\beta}{3 p}-\tau, & 1 \leq p<2, \\ \frac{1}{2}-\tau-\varepsilon, & p=2, \\ \frac{1}{p}-\tau, & p>2,\end{cases}
$$

where $\varepsilon>0$ can be taken arbitrarily small.

Proof. It follows from Proposition 6.1 that condition (1.9) is satisfied. To show that condition (1.10) holds, we use the estimates for $L^{q}$-norms of $\left\{\psi_{k}\right\}$ from Proposition 6.3 with the weight $w(x)=\left(1+x^{2}\right)^{\tau / 2}$. The rest is straightforward, like the proof of [29, Thm.3],

$$
\begin{aligned}
\left|b_{V}\left(\psi_{m}, \psi_{n}\right)\right| & \leq \int_{\mathbb{R}} w^{-1}|V| w\left|\psi_{m}\right|\left|\psi_{n}\right| \mathrm{d} x \leq\left\|w^{-1}|V|\right\|_{L^{p}(\mathbb{R})}\left\|w \psi_{m} \psi_{n}\right\|_{L^{q}(\mathbb{R})} \\
& \leq C\left\|w^{\frac{1}{2}} \psi_{m}\right\|_{L^{2 q}(\mathbb{R})}\left\|w^{\frac{1}{2}} \psi_{n}\right\|_{L^{2 q}(\mathbb{R})},
\end{aligned}
$$

where $1 / p+1 / q=1$. Condition (1.10) is satisfied due to (6.39) and (6.41).

Putting together Theorems 6.4 and 3.4 we obtain the following claim on the eigensystem of perturbed Schrödinger operators $T$.

Corollary 6.5. Let $A$ be as in (6.1) and $V \in L(p, \tau)$ with $p \in[1, \infty]$ and $\tau \geq 0$ satisfying (6.69). Then the eigensystem of $T$, being the form sum of these $A$ and $V$, contains a Riesz basis of $L^{2}(\mathbb{R})$.

6.3.1. $L^{1}$-potentials with a controlled decay. We consider a potential $V \in L^{1}(\mathbb{R})$ with the decay $|x|^{-1-\varepsilon}$ for some $\varepsilon>0$ at infinity. More precisely, we suppose that

$$
\begin{aligned}
& V=V_{1}+V_{2}, \\
& V_{1} \in L^{1}(\mathbb{R}), \operatorname{supp} V_{1} \text { is compact, } \\
& \exists \varepsilon>0,\left(1+x^{2}\right)^{\frac{1+\varepsilon}{2}} V_{2}(x) \in L^{\infty}(\mathbb{R}) .
\end{aligned}
$$

Since such a $V$ is integrable on $\mathbb{R}$, it follows from Theorem 6.4 that the form associated with this $V$ satisfies condition (1.10) with $\alpha=(4-\beta) /(6(\beta+2))$. We show in the following that the latter improves if $(6.72)$ is satisfied, moreover, we derive a more convenient formula for the first correction $\lambda_{n}^{(1)}$ from Theorem 3.2.

Theorem 6.6. Let $Q$ satisfy Assumption I, let A be Schrödinger operator from (6.1) and let $V$ satisfy (6.72). Then the form $b_{V}$ from (6.68) satisfy condition (1.10) with

$$
\alpha=\frac{1}{\beta+2} .
$$

Moreover, the terms $\left\{\lambda_{n}^{(1)}\right\}$ from Theorem 3.2 for $T$ being the form sum of $A$ and $\checkmark$ satisfy

$$
\lambda_{n}^{(1)}=\frac{1}{\Omega_{\beta}^{\prime}}\left(\frac{\pi}{\Omega_{\beta}} n\right)^{-\frac{2}{\beta+2}} \int_{\mathbb{R}} V(x) \mathrm{d} x+o\left(n^{-\frac{2}{\beta+2}}\right), \quad n \rightarrow \infty,
$$

where $\Omega_{\beta}, \Omega_{\beta}^{\prime}$ are as in (6.14).

Proof. We show below that

$$
\int_{0}^{\infty} V u^{2} \mathrm{~d} x=\frac{\pi}{\mu^{\frac{1}{2}}} \int_{0}^{\infty} V \mathrm{~d} x(1+o(1)), \quad \mu \rightarrow \infty .
$$

Hence, using that $u^{2}$ are even, (6.34), (6.26) and

$$
\left|b_{V}\left(\psi_{m}, \psi_{n}\right)\right| \leq\left(\int_{\mathbb{R}}|V|\left|\psi_{m}\right|^{2}\right)^{\frac{1}{2}}\left(\int_{\mathbb{R}}|V|\left|\psi_{n}\right|^{2}\right)^{\frac{1}{2}},
$$


we obtain that $b_{V}$ satisfies condition (1.10) with $\alpha$ in (6.73). The claim (6.74) follows from (3.31), (6.75), (6.34) and (6.12).

It remains to prove the key step (6.75). We analyze the integral in (6.75) separately in several regions.

- $0<x<x_{\sqrt{\mu}}$ : As $\mu \rightarrow \infty$, we have

$$
\frac{1}{(\mu-Q(x))^{\frac{1}{2}}}-\frac{1}{\mu^{\frac{1}{2}}}=\frac{Q(x)}{\mu^{\frac{1}{2}}(\mu-Q(x))^{\frac{1}{2}}\left(\mu^{\frac{1}{2}}+(\mu-Q(x))^{\frac{1}{2}}\right)}=\mathcal{O}\left(\mu^{-1}\right) .
$$

Hence formula (6.29) and $V \in L^{1}(\mathbb{R})$ give

$$
\int_{0}^{x \sqrt{\mu}} V u^{2} \mathrm{~d} x=\frac{\pi}{\mu^{\frac{1}{2}}} \int_{0}^{x \sqrt{\mu}} V\left(1+\sin 2 \zeta+R_{1}(\zeta)\right) \mathrm{d} x+\mathcal{O}\left(\mu^{-1}\right), \quad \mu \rightarrow \infty .
$$

Next we show that

$$
\int_{0}^{x \sqrt{\mu}} V \sin 2 \zeta \mathrm{d} x=o(1), \quad \mu \rightarrow \infty
$$

and

$$
\int_{0}^{x \sqrt{\mu}} V R_{1}(\zeta) \mathrm{d} x=\mathcal{O}\left(\mu^{-\frac{1}{2}} x_{\mu}^{-1}\right), \quad \mu \rightarrow \infty,
$$

therefore

$$
\int_{0}^{x \sqrt{\mu}} V u^{2} \mathrm{~d} x=\frac{\pi}{\mu^{\frac{1}{2}}} \int_{0}^{x \sqrt{\mu}} V \mathrm{~d} x+o\left(\mu^{-\frac{1}{2}}\right), \quad \mu \rightarrow \infty .
$$

For any $\varepsilon>0$, find $V_{\varepsilon} \in C_{0}^{\infty}(\mathbb{R})$ such that $\left\|V-V_{\varepsilon}\right\|_{L^{1}(\mathbb{R})}<\varepsilon$. Then

$$
\left|\int_{0}^{x_{\sqrt{\mu}}} V \sin 2 \zeta \mathrm{d} x\right| \leq \varepsilon\|V\|_{L^{1}(\mathbb{R})}+\left|\int_{0}^{x_{\sqrt{\mu}}} V_{\varepsilon} \sin 2 \zeta \mathrm{d} x\right| .
$$

The integration by parts yields

$$
\begin{aligned}
\int_{0}^{x_{\sqrt{\mu}}} V_{\varepsilon} \sin 2 \zeta \mathrm{d} x= & -\frac{\cos (2 \zeta(0)) V_{\varepsilon}(0)}{2} \mu^{-\frac{1}{2}} \\
& -\int_{0}^{x_{\sqrt{\mu}}} \frac{\cos 2 \zeta}{2}\left(\frac{V_{\varepsilon}^{\prime}}{(\mu-Q)^{\frac{1}{2}}}+\frac{V_{\varepsilon} Q^{\prime}}{2(\mu-Q)^{\frac{3}{2}}}\right) \mathrm{d} x \\
= & \mathcal{O}\left(\mu^{-\frac{1}{2}}\right)+\mathcal{O}\left(a_{\mu} \mu^{-\frac{3}{2}}\right)=\mathcal{O}\left(\mu^{-\frac{1}{2}}\right), \quad \mu \rightarrow \infty,
\end{aligned}
$$

where we use that $V_{\varepsilon}\left(x_{\sqrt{\mu}}\right)=0$ for all sufficiently large $\mu$ and (6.2) in the last step. Since $\varepsilon>0$ is arbitrary, we conclude with (6.79).

Since $\left|R_{1}(\zeta)\right|<1 / \zeta$, see (6.29) and below, and $\zeta$ is decreasing, we have

$$
\int_{0}^{x_{\sqrt{\mu}}}\left|V R_{1}(\zeta)\right| \mathrm{d} x \leq \frac{\|V\|_{L^{1}(\mathbb{R})}}{\zeta\left(x_{\sqrt{\mu}}\right)} \leq \frac{\|V\|_{L^{1}(\mathbb{R})}}{\left(\mu-\mu^{\frac{1}{2}}\right)^{\frac{1}{2}}\left(x_{\mu}-x_{\sqrt{\mu}}\right)} .
$$

By the mean value theorem,

$$
x_{\mu}-x_{\sqrt{\mu}}=Q^{-1}(\mu)-Q^{-1}\left(\mu^{\frac{1}{2}}\right) \geq \frac{\mu-\mu^{\frac{1}{2}}}{a_{\mu}},
$$

thus (6.80) follows from (6.84), (6.85) and (6.2).

- $x_{\sqrt{\mu}}<x<x_{\frac{\mu}{2}}$ : From (6.30) and $V \in L^{1}(\mathbb{R})$, we have

$$
\begin{aligned}
\int_{x_{\sqrt{\mu}}}^{x \frac{\mu}{2}}|V| u^{2} \mathrm{~d} x & \leq A_{1}^{2} \int_{x_{\sqrt{\mu}}}^{x_{\frac{\mu}{2}}} \frac{|V| \mathrm{d} x}{(\mu-Q)^{\frac{1}{2}}} \\
& =\mathcal{O}\left(\mu^{-\frac{1}{2}}\right) \int_{x_{\sqrt{\mu}}}^{x \frac{\mu}{2}}|V| \mathrm{d} x=o\left(\mu^{-\frac{1}{2}}\right), \quad \mu \rightarrow \infty .
\end{aligned}
$$


- $x_{\frac{\mu}{2}}<x<x_{\frac{3}{2} \mu}$ : Since the support of $V_{1}$ is compact, only the integral with $V_{2}$ contributes for large $\mu$. Due to the controlled decay of $V_{2}$, see (6.72), we have

$$
\int_{x_{\frac{\mu}{2}}}^{x_{\frac{3}{2} \mu}}|V| u^{2} \mathrm{~d} x=\mathcal{O}\left(x_{\mu}^{-1-\varepsilon}\right) \int_{x_{\frac{\mu}{2}}}^{x_{\frac{3}{2} \mu}} u^{2} \mathrm{~d} x, \quad \mu \rightarrow \infty .
$$

The integral of $u^{2}$ is estimated in the proof of Proposition 6.3, namely,

$$
\left(\int_{x_{\frac{\mu}{2}}}^{x_{\mu}-\delta}+\int_{x_{\mu}-\delta}^{x_{\mu}+\delta_{1}}+\int_{x_{\mu}+\delta_{1}}^{x_{\frac{3}{2} \mu}}\right) u^{2} \mathrm{~d} x=\mathcal{O}\left(a_{\mu}^{-1} \mu^{\frac{1}{2}}\right)+\mathcal{O}\left(a_{\mu}^{-\frac{2}{3}}\right), \quad \mu \rightarrow \infty .
$$

Thus, we get from (6.7) that

$$
\int_{x_{\frac{\mu}{2}}}^{x_{\frac{3}{2} \mu}}|V| u^{2} \mathrm{~d} x=\mathcal{O}\left(\mu^{-\frac{1}{2}} x_{\mu}^{-\varepsilon}\right), \quad \mu \rightarrow \infty .
$$

- $x_{\frac{3}{2} \mu}<x$ : Again, there is no contribution of $V_{1}$ for large $\mu$ and (6.72), (6.30) and (6.61) yield (with some $C>0$, see also (6.62))

$$
\int_{x_{\frac{3}{2} \mu}}^{\infty}|V| u^{2} \mathrm{~d} x=\mathcal{O}\left(x_{\mu}^{-1-\varepsilon} e^{-C \mu^{\frac{1}{2}} x_{\mu}}\right), \quad \mu \rightarrow \infty .
$$

Putting all these estimates together, we indeed get (6.75).

Remark 6.7. For special choice $Q(x)=|x|^{\beta}, \beta \geq 2$, we obtain more precise asymptotics for $\left\{\mu_{n}\right\}$ from (6.10). Thus we can conclude further that, for $V$ as in (6.72), the eigenvalues $\left\{\lambda_{n}\right\}$ of $T$ satisfy as $n \rightarrow \infty$

$$
\lambda_{n}=\left(\frac{\pi}{\Omega_{\beta}}\left(n+\frac{1}{2}\right)\right)^{\frac{2 \beta}{\beta+2}}+\frac{1}{\Omega_{\beta}^{\prime}}\left(\frac{\pi}{\Omega_{\beta}} n\right)^{-\frac{2}{\beta+2}} \int_{\mathbb{R}} V(x) \mathrm{d} x+o\left(n^{-\frac{2}{\beta+2}}\right) .
$$

When taking formally the limit $\beta \rightarrow+\infty$, the correction (6.74) due to $V$ becomes (5.20) with $l=1$; notice that the formula (5.20) is valid also for the perturbation of $-\mathrm{d}^{2} / \mathrm{d} x^{2}$ with Dirichlet boundary conditions.

6.4. Perturbations by singular potentials. Let $V \in W^{-s, 2}(\mathbb{R})$ with some $s \geq$ 0 , so

$$
\exists C>0, \exists s \geq 0, \forall \phi \in W^{1,2}(\mathbb{R}),|(V, \phi)| \leq C\|\phi\|_{W^{1,2}(\mathbb{R})}^{s}\|\phi\|^{1-s},
$$

and define the form

$$
b_{V}(\phi, \psi):=(V, \phi \bar{\psi}), \quad \phi, \psi \in \operatorname{Dom}(a) .
$$

An extension of Theorem 6.4 for singular potentials is Theorem 6.9 below, where sufficient conditions on $V$ so that $b_{V}$ satisfies (1.10) are stated. In the proof, the following estimates of $L^{q}$-norms of $\left\{\psi_{k}^{\prime}\right\}$ are used.

Lemma 6.8. Let $Q$ satisfy Assumption I with (6.3) replaced by (6.11), let $\left\{\mu_{k}\right\}$ be the eigenvalues of $A$ and let $\left\{\psi_{k}\right\}$ be as in (6.26). Then

$$
\left\|\psi_{k}^{\prime}\right\|_{L^{q}(\mathbb{R})}=\mathcal{O}\left(\mu_{k}^{\frac{1}{2}}\left\|\psi_{k}\right\|_{L^{q}(\mathbb{R})}\right), \quad k \rightarrow \infty .
$$

Proof. By the Gagliardo-Nirenberg interpolation inequality with $\alpha=1 / 2$, see [31, Lecture II], and since $\psi_{k}$ is an eigenfunction of $A$, we get

$$
\begin{aligned}
\left\|\psi_{k}^{\prime}\right\|_{L^{q}(\mathbb{R})} & \leq C\left\|\psi_{k}^{\prime \prime}\right\|_{L^{q}(\mathbb{R})}^{\frac{1}{2}}\left\|\psi_{k}\right\|_{L^{q}(\mathbb{R})}^{\frac{1}{2}} \\
& \leq C\left(\mu_{k}^{\frac{1}{2}}\left\|\psi_{k}\right\|_{L^{q}(\mathbb{R})}^{\frac{1}{2}}+\left\|Q^{\frac{1}{2}} \psi_{k}\right\|_{L^{q}(\mathbb{R})}^{\frac{1}{2}}\right)\left\|\psi_{k}\right\|_{L^{q}(\mathbb{R})}^{\frac{1}{2}} .
\end{aligned}
$$

Finally, $Q^{\frac{1}{2}}$ satisfies the conditions on the weight $w$ in Proposition 6.3 and so (6.94) follows from (6.95) and (6.39) with $w=Q^{\frac{1}{2}}$. 
Theorem 6.9. Let $Q$ satisfy Assumption I and let $A$ be Schrödinger operator from (6.1). If $V \in W^{-s, 2}(\mathbb{R})$ with

$$
0 \leq s<\frac{\beta-1}{2 \beta}
$$

then $A$ and the form $b_{V}$ from (6.93) satisfy condition (1.10) with

$$
\gamma=\frac{2 \beta}{\beta+2}, \quad \alpha=\frac{1-2 \beta s}{2(\beta+2)} .
$$

If the support of $V$ is compact and

$$
0 \leq s<\frac{1}{2}
$$

then $A$ and the form $b_{V}$ from (6.93) satisfy condition (1.10) with

$$
\gamma=\frac{2 \beta}{\beta+2}, \quad \alpha=\frac{1-\beta s}{\beta+2} .
$$

Proof. The letter $C$ denotes a constant, which can vary in every step, however, it is independent of $m$ and $n$. We employ (6.92), Hölder inequality, (6.94) with $q=4$ and finally $(6.12),(6.41)$

$$
\begin{aligned}
\left|b_{V}\left(\psi_{m}, \psi_{n}\right)\right| & \leq C\left(\left\|\psi_{m}^{\prime} \psi_{n}\right\|^{s}+\left\|\psi_{n}^{\prime} \psi_{m}\right\|^{s}+\left\|\psi_{m} \psi_{n}\right\|^{s}\right)\left\|\psi_{m} \psi_{n}\right\|^{1-s} . \\
& \leq C\left(\mu_{m}^{\frac{s}{2}}+\mu_{n}^{\frac{s}{2}}+1\right)\left\|\psi_{m}\right\|_{L^{4}(\mathbb{R})}\left\|\psi_{n}\right\|_{L^{4}(\mathbb{R})} \\
& \leq C\left(\mu_{m} \mu_{n}\right)^{\frac{s}{2}}\left\|\psi_{m}\right\|_{L^{4}(\mathbb{R})}\left\|\psi_{n}\right\|_{L^{4}(\mathbb{R})} \\
& \leq C(m n)^{\frac{2 \beta s-1}{2(\beta+2)}} \log m \log n .
\end{aligned}
$$

Thus condition (1.10) is satisfied with $\alpha$ as in (6.97).

If the support of $V$ is compact, we use the pointwise bounds for $\left|\psi_{k}\right|$ and $\left|\psi_{k}^{\prime}\right|$, see (6.35), (6.36). Similarly as above, we obtain from (6.92) that

$$
\left|b_{V}\left(\psi_{m}, \psi_{n}\right)\right| \leq C(m n)^{\frac{\beta s-1}{\beta+2}}
$$

and so condition (1.10) is satisfied with $\alpha$ as in (6.99).

Notice that for $\beta \rightarrow+\infty$ in (6.96), we recover the condition $s<1 / 2$ derived in Section 5.3.4 for perturbations of $-\mathrm{d}^{2} / \mathrm{d} x^{2}$ on finite interval.

Corollary 6.10. Let $A$ be as in (6.1) and $V \in W^{-s, 2}(\mathbb{R})$ with s satisfying (6.96) or (6.98) if the support of $V$ is compact. Then the eigensystem of $T$, being the form sum of these $A$ and $V$, contains a Riesz basis of $L^{2}(\mathbb{R})$.

Finally, we consider the perturbation of $A$ by $\delta$-interactions like in Section 5.3.2, see also (1.11). Let

$$
b_{\delta}^{\infty}[\psi]:=\sum_{k \in \mathbb{Z}} \nu_{k}\left|\psi\left(x_{k}\right)\right|^{2}, \quad\left\{\nu_{k}\right\} \in \ell^{1}(\mathbb{Z}), \quad\left\{x_{k}\right\} \subset \mathbb{R}, \quad \psi \in \operatorname{Dom}(a) .
$$

It follows from (6.37) that

$$
\left|b_{\delta}^{\infty}\left(\psi_{m}, \psi_{n}\right)\right| \leq C\|\nu\|_{\ell^{1}(\mathbb{Z})}(m n)^{\frac{\beta-4}{6(\beta+2)}},
$$

thus $A$ and $b_{\delta}^{\infty}$ satisfy condition (1.10) if $\beta>1$. Hence, by Theorem 3.4, the eigensystem of $T$, being the form sum of $A$ in (6.1) and $b_{\delta}^{\infty}$ in (6.102), contains a Riesz basis of $L^{2}(\mathbb{R})$. 


\section{REFERENCES}

[1] Adduci, J., Duakov, P., And Mityagin, B. Convergence Radii for Eigenvalues of TriDiagonal Matrices. Lett. Math. Phys. 91 (2010), 45-60.

[2] Adduci, J., And Mityagin, B. Eigensystem of an $L^{2}$-perturbed harmonic oscillator is an unconditional basis. Cent. Eur. J. Math. 10 (2012), 569-589.

[3] Adduci, J., And Mityagin, B. Root System of a Perturbation of a Selfadjoint Operator with Discrete Spectrum. Integral Equations Operator Theory 73 (2012), 153-175.

[4] Agranovich, M. S. On series in root vectors of operators defined by forms with a selfadjoint principal part. Funktsional'nyı Analiz i ego Prilozheniya 28 (1994), 1-21, 95. Translation in Functional Analysis and Its Applications 28 (1994), 151-167.

[5] Davies, E. B. Semi-Classical States for Non-Self-Adjoint Schrödinger Operators. Comm. Math. Phys. 200 (1999), 35-41.

[6] Davies, E. B. Wild spectral behaviour of anharmonic oscillators. Bull. Lond. Math. Soc. 32 (2000), 432-438.

[7] Davies, E. B., And KuiJlaars, A. B. J. Spectral asymptotics of the non-self-adjoint harmonic oscillator. J. London Math. Soc. 70 (2004), 420-426.

[8] Djakov, P., And Mityagin, B. Bari-Markus property for Riesz projections of Hill operators with singular potentials. In Functional analysis and complex analysis, vol. 481 of Contemp. Math. Amer. Math. Soc., Providence, RI, 2009, pp. 59-80.

[9] Djakov, P., AND Mityagin, B. Spectral gaps of Schrödinger operators with periodic singular potentials. Dyn. Partial Differ. Equ. 6 (2009), 95-165.

[10] Djakov, P., And Mityagin, B. Fourier method for one-dimensional Schrödinger operators with singular periodic potentials. In Topics in operator theory. Vol. 2. Systems and mathematical physics, vol. 203 of Oper. Theory Adv. Appl. Birkhäuser Verlag, Basel, 2010, pp. 195236.

[11] Djakov, P., And Mityagin, B. Equiconvergence of spectral decompositions of HillSchrödinger operators. J. Differential Equations 255 (2013), 3233-3283.

[12] Dunford, N., And Schwartz, J. T. Linear Operators. John Wiley \& Sons, Inc., New York, 1988.

[13] Elton, D. M. Asymptotics for the Eigenvalues of the Harmonic Oscillator with a QuasiPeriodic Perturbation. arXiv:math/0312110, 2003.

[14] Elton, D. M. The Bethe-Sommerfield conjecture for the 3-dimensional periodic Landau operator. Rev. Math. Phys. 16 (2004), 1259-1290.

[15] Giertz, M. On the solutions in $L^{2}(-\infty, \infty)$ of $y^{\prime \prime}+(\lambda-q(x)) y=0$ when $q$ is rapidly increasing. Proc. London Math. Soc. 14 (1964), 53-73.

[16] Gohberg, I. C., And Krein, M. G. Introduction to the Theory of Linear Nonselfadjoint Operators in Hilbert Space. AMS, Providence, 1969.

[17] Halmos, P. R., And Sunder, V. S. Bounded integral operators on $L^{2}$ spaces. Springer-Verlag, Berlin, 1978.

[18] Henry, R. Spectral instability of some non-selfadjoint anharmonic oscillators. C. R. Math. Acad. Sci. Paris 350 (2012), 1043-1046.

[19] Henry, R. Spectral instability for even non-selfadjoint anharmonic oscillators. J. Spec. Theory 4 (2014), 349-364

[20] Henry, R. Spectral Projections of the Complex Cubic Oscillator. Ann. Henri Poincaré 15 (2014), 2025-2043.

[21] Kato, T. Perturbation theory for linear operators. Springer-Verlag, Berlin, 1995.

[22] Krasnoselskit, M. A., Zabreiko, P. P., Pustylnik, E. I., And Sobolevskit, P. E. Integral operators in spaces of summable functions. Noordhoff International Publishing, Leiden, 1976.

[23] Krejčiřík, D., Siegl, P., Tater, M., And Viola, J. Pseudospectra in non-Hermitian quantum mechanics. J. Math. Phys. 56 (2015), 103513.

[24] Markus, A. Introduction to the spectral theory of polynomial operator pencils. American Mathematical Society, Providence, 1988.

[25] Mikhajlov, V. Riesz bases in $\mathcal{L}_{2}(0,1)$. Sov. Math. Dokl. 3 (1962), 851-855.

[26] Mityagin, B. The spectrum of a Harmonic Oscillator Operator Perturbed by Point Interactions. arXiv:1407.4153, 2014

[27] Mityagin, B. The Spectrum of a Harmonic Oscillator Operator Perturbed by Point Interactions. Int. J. Theor. Phys. 54 (2015), 4068-4085.

[28] Mityagin, B. The spectrum of a harmonic oscillator operator perturbed by $\delta$-interactions. Integral Equations Operator Theory 85 (2016), 451-495.

[29] Mityagin, B., And Siegl, P. Root system of singular perturbations of the harmonic oscillator type operators. Lett. Math. Phys. 106 (2016), 147-167. 
[30] Mityagin, B., Siegl, P., And Viola, J. Differential operators admitting various rates of spectral projection growth. J. Funct. Anal. 272 (2017), 3129-3175.

[31] Nirenberg, L. On elliptic partial differential equations. Ann. Scuola Norm. Sup. Pisa 13 (1959), 115-162.

[32] Reed, M., And Simon, B. Methods of Modern Mathematical Physics, Vol. 4: Analysis of Operators. Academic Press, New York-London, 1978.

[33] Schur, I. Bemerkungen zur Theorie der Beschrankten Bilinearformen mit unendlich vielen Veranderlichten. J. Reine Angew. Math. 140 (1911), 1-28.

[34] Shkalikov, A. On the basis property of root vectors of a perturbed self-adjoint operator. Proc. Steklov Inst. Math. 269 (2010), 284-298.

[35] Shkalikov, A. Eigenvalue asymptotics of perturbed self-adjoint operators. Methods Funct. Anal. Topology 18 (2012), 79-89.

[36] Siegl, P., AND KRejČIŕíK, D. On the metric operator for the imaginary cubic oscillator. Phys. Rev. D 86 (2012), 121702(R).

[37] Thangavelu, S. Lectures on Hermite and Laguerre expansions. Princeton University Press, Princeton, 1993.

[38] Titchmarsh, E. C. Eigenfunction expansions associated with second-order differential equations. Part II. Clarendon Press, Oxford, 1958.

[39] Titchmarsh, E. C. Eigenfunction expansions associated with second-order differential equations. Part I. Clarendon Press, Oxford, 1962.

(Boris Mityagin) Department of Mathematics, The Ohio State University, 231 West 18th Ave, Columbus, OH 43210, USA

E-mail address: mityagin.1@osu.edu

(Petr Siegl) Mathematical Institute, University of Bern, Alpeneggstr. 22, 3012 Bern, Switzerland \& On leave from Nuclear Physics Institute CAS, 25068 Řž̌, Czech Republic E-mail address: petr.siegl@math.unibe.ch 